\title{
On the linear independence constraint qualification in disjunctive programming
}

\author{
Patrick Mehlitz*
}

July 1, 2019

\begin{abstract}
Mathematical programs with disjunctive constraints (MPDCs for short) cover several different problem classes from nonlinear optimization including complementarity-, vanishing-, cardinality-, and switching-constrained optimization problems. In this paper, we introduce an abstract but reasonable version of the prominent linear independence constraint qualification which applies to MPDCs. Afterwards, we derive first- and second-order optimality conditions for MPDCs under validity of this constraint qualification based on so-called strongly stationary points. Finally, we apply our findings to some popular classes of disjunctive programs and compare the obtained results to those ones available in the literature. Particularly, new second-order optimality conditions for mathematical programs with switching constraints are by-products of our approach.
\end{abstract}

Keywords: Constraint qualifications, Disjunctive programming, Linear independence constraint qualification, Strong stationarity, Second-order optimality conditions MSC: 90C30, 90C33

\section{Introduction}

In this paper, so-called mathematical programs with disjunctive constraints (MPDCs) are studied. These are optimization problems of the form

$$
\begin{aligned}
& f(x) \rightarrow \min \\
& F(x) \in D
\end{aligned}
$$

where $f: \mathbb{R}^{n} \rightarrow \mathbb{R}$ as well as $F: \mathbb{R}^{n} \rightarrow \mathbb{R}^{m}$ are twice continuously differentiable and $D:=\bigcup_{i=1}^{r} D_{i}$ is the finite union of given polyhedral sets $D_{1}, \ldots, D_{r} \subset \mathbb{R}^{m}$. Recall that

\footnotetext{
*Brandenburgische

Technische

Universität

Cottbus-Senftenberg,

Insti-

tute of Mathematics, 03046 Cottbus, Germany, mehlitz@b-tu.de, https://www.b-tu.de/fg-optimale-steuerung/team/dr-patrick-mehlitz, ORCID: 0000-00029355-850X
} 
a set is called polyhedral whenever it can be represented as the intersection of finitely many half spaces. We use $X:=\left\{x \in \mathbb{R}^{n} \mid F(x) \in D\right\}$ in order to denote the feasible set of (MPDC). Clearly, choosing $r:=1$ and $D:=\mathbb{R}_{-}^{p} \times\left\{0^{q}\right\}$ with $p, q \in \mathbb{N}$ and $m:=p+q$, any standard nonlinear program is a disjunctive program, see Example 3.2. However, it is well known that the model (MPDC) covers mathematical programs with complementarity constraints (MPCCs), see Luo et al. [1996], mathematical programs with vanishing constraints (MPVCs), see Achtziger and Kanzow [2008], cardinality-constrained mathematical programs (CCMPs), see Pan et al. [2017], and mathematical programs with switching constraints (MPSCs), see Mehlitz [2019], as well. Note that all these problem classes which frequently arise from the mathematical modeling of real-world applications suffer from an inherent lack of regularity. That is why huge effort has been put into the derivation of problem-tailored stationarity notions and constraint qualifications. However, it is clear that any theoretical result which can be derived for the generalized model (MPDC) has a corresponding counterpart for MPCCs, MPVCs, CCMPs, and MPSCs. This observation justifies the theoretical investigation of (MPDC). First ideas on how to study disjunctive structures in nonlinear optimization are presented in Scholtes [2004]. Stationarity notions and constraint qualifications for (MPDC) can be found in [Benko and Gfrerer, 2018, Flegel et al., 2007, Gfrerer, 2014]. Particularly, second-order necessary and sufficient optimality conditions for disjunctive programs are derived in Gfrerer [2014] with the aid of the celebrated directional limiting calculus, see Gfrerer [2013] as well. Checking [Gfrerer, 2014, Theorems 3.3, 3.17], one can observe that in contrast to classical second-order optimality conditions, the appearing set of multipliers depends on the choice of the particular critical direction.

In this paper, we are going to state an MPDC-tailored version of the linear independence constraint qualification (LICQ) and study its inherent properties. Furthermore, we derive second-order necessary optimality conditions for (MPDC) under validity of this constraint qualification in a completely elementary way using second-order tangent sets. Thus, our approach is related to techniques which were used in Bonnans and Shapiro [2000], Christof and Wachsmuth [2018], Penot [1998], Rockafellar and Wets [1998] to derive second-order conditions for mathematical programs. On the other hand, we present a result which shows the isolatedness of strongly stationary points of (MPDC) where the problem-tailored version of LICQ and a suitable second-order sufficient condition hold. This generalizes some corresponding results for MPCCs, see Guo et al. [2013], and CCMPs, see [Bucher and Schwartz, 2018, Corollary 3.3]. Afterwards, we apply our findings to several instances of disjunctive programming. In particular, new second-order optimality conditions for MPSCs will be derived.

The remaining parts of this paper are structured as follows: In Section 2, we comment on the notation used in this manuscript and introduce all the necessary tools from variational analysis which are exploited later. Furthermore, some preliminary results are provided. Section 3 is dedicated to the derivation of an MPDC-tailored version of the linear independence constraint qualification. Some consequences of the validity of this regularity condition are presented. Second-order optimality conditions for (MPDC) are the topic of interest in Section 4. First, it will be shown that a second-order necessary optimality condition holds at the local minimizers of (MPDC) where our new 
constraint qualification is valid. Afterwards, a second-order sufficient optimality condition for (MPDC) will be derived. Subsequently, we show that this condition together with the problem-tailored version of LICQ implies that the underlying strongly stationary point of interest is in a certain sense locally isolated. In Section 5, we apply the derived theory to MPCCs, MPVCs, as well as CCMPs and compare our findings to available results from the literature, see Scheel and Scholtes [2000], Hoheisel and Kanzow [2007], Bucher and Schwartz [2018]. Furthermore, we obtain new second-order optimality conditions for MPSCs. Some final remarks close the paper in Section 6.

\section{Preliminaries}

\subsection{Basic notation}

Throughout this paper, $x \cdot y$ is used to denote the common Euclidean inner product of two vectors $x, y \in \mathbb{R}^{n}$. We equip $\mathbb{R}^{n}$ with the Euclidean norm $\|\cdot\|_{2}$. The zero vector in $\mathbb{R}^{n}$ will be denoted by $0^{n}$ while 0 is used to represent the scalar zero. For $\varepsilon>0$ and some $\bar{x} \in \mathbb{R}^{n}, \mathbb{B}^{\varepsilon}(\bar{x}):=\left\{x \in \mathbb{R}^{n} \mid\|x-\bar{x}\|_{2} \leq \varepsilon\right\}$ denotes the closed $\varepsilon$-ball around $\bar{x}$. Similarly, $\mathbb{U}^{\varepsilon}(\bar{x}):=\left\{x \in \mathbb{R}^{n} \mid\|x-\bar{x}\|_{2}<\varepsilon\right\}$ represents the open $\varepsilon$-ball around $\bar{x}$. Frequently, we will make use of the sets $\mathbb{R}_{+}:=\{t \in \mathbb{R} \mid t \geq 0\}$ and $\mathbb{R}_{-}:=\{t \in \mathbb{R} \mid t \leq 0\}$. For a given nonempty set $A \subset \mathbb{R}^{n}$, we exploit $\operatorname{cl} A$, cone $A, \operatorname{conv} A, \overline{\operatorname{conv}} A$, and $\operatorname{span} A$ in order to represent the closure of $A$, the conic hull of $A$, the convex hull of $A$, the closed convex hull of $A$, and the span of $A$ (i.e. the smallest subspace of $\mathbb{R}^{n}$ comprising $A$ ), respectively. We use $\operatorname{dist}(x, A):=\inf \left\{\|z-x\|_{2} \mid z \in A\right\}$ to represent the distance of $x \in \mathbb{R}^{n}$ to $A$. Finally, the Cartesian product $A \times B$ of two sets $A \subset \mathbb{R}^{n}$ and $B \subset \mathbb{R}^{m}$ will be interpreted as a subset of $\mathbb{R}^{n+m}$.

Recall that a set-valued mapping $\Psi: \mathbb{R}^{n} \rightrightarrows \mathbb{R}^{m}$, i.e. a mapping which assigns to each $x \in \mathbb{R}^{n}$ a (possibly empty) set $\Psi(x) \subset \mathbb{R}^{m}$, is called metrically subregular at some point $(\bar{x}, \bar{y}) \in\left\{(x, y) \in \mathbb{R}^{n} \times \mathbb{R}^{m} \mid y \in \Psi(x)\right\}$ if there are constants $\kappa>0$ and $\varepsilon>0$ such that

$$
\forall x \in \mathbb{U}^{\varepsilon}(\bar{x}): \quad \operatorname{dist}\left(x, \Psi^{-1}(\bar{y})\right) \leq \kappa \operatorname{dist}(\bar{y}, \Psi(x))
$$

holds true. Here, $\Psi^{-1}: \mathbb{R}^{m} \rightrightarrows \mathbb{R}^{n}$ denotes the inverse set-valued mapping associated with $\Psi$ which is defined by $\Psi^{-1}(y):=\left\{x \in \mathbb{R}^{n} \mid y \in \Psi(x)\right\}$ for all $y \in \mathbb{R}^{m}$. It is easily seen that $\Psi$ is metrically subregular at $(\bar{x}, \bar{y})$ if and only if $\Psi^{-1}$ possesses the so-called calmness property at $(\bar{y}, \bar{x})$, see e.g. Henrion and Outrata [2005].

For a twice continuously differentiable mapping $P: \mathbb{R}^{n} \rightarrow \mathbb{R}^{m}, \nabla P(x) \in \mathbb{R}^{m \times n}$ denotes its Jacobian at $x \in \mathbb{R}^{n}$. In the particular case $m=1$, the gradient $\nabla P(x)$ will be interpreted as a column vector. Furthermore, we set

$$
\forall x \in \mathbb{R}^{n} \forall d, h \in \mathbb{R}^{n}: \quad \nabla^{2} P(x)[d, h]:=\left(\begin{array}{c}
d^{\top} \nabla^{2} P_{1}(x) h \\
\vdots \\
d^{\top} \nabla^{2} P_{m}(x) h
\end{array}\right)
$$

where $P_{1}, \ldots, P_{m}: \mathbb{R}^{n} \rightarrow \mathbb{R}$ are the component mappings associated with $P$ while the matrices $\nabla^{2} P_{1}(x), \ldots, \nabla^{2} P_{m}(x)$ are their respective Hessians at $x \in \mathbb{R}^{n}$. 


\subsection{Variational analysis}

Here, we introduce the notions of variational analysis which are necessary in order to carry out our later considerations. For terminology and notation, we mainly follow Aubin and Frankowska [2009], Bonnans and Shapiro [2000], Rockafellar and Wets [1998].

\subsubsection{Polars and annihilators}

For a nonempty set $A \subset \mathbb{R}^{n}$, the polar cone and the annihilator of $A$ are given as stated below:

$$
A^{\circ}:=\left\{y \in \mathbb{R}^{n} \mid \forall x \in A: x \cdot y \leq 0\right\}, \quad A^{\perp}:=\left\{y \in \mathbb{R}^{n} \mid \forall x \in A: x \cdot y=0\right\} .
$$

Obviously, $A^{\circ}$ is a closed, convex cone and one has $A^{\perp}=A^{\circ} \cap(-A)^{\circ}$, i.e. $A^{\perp}$ is a subspace of $\mathbb{R}^{n}$. For any two sets $A, B \subset \mathbb{R}^{n}$, one easily obtains $(A \cup B)^{\circ}=A^{\circ} \cap B^{\circ}$ as well as $(A \cup B)^{\perp}=A^{\perp} \cap B^{\perp}$. For a cone $C \subset \mathbb{R}^{n}$, the so-called bipolar theorem, see [Rockafellar and Wets, 1998, Corollary 6.21], shows $C^{\circ 0}=\overline{\operatorname{conv}} C$. Furthermore, the polarization rule $\left(C_{1}+C_{2}\right)^{\circ}=C_{1}^{\circ} \cap C_{2}^{\circ}$ follows for any two cones $C_{1}, C_{2} \subset \mathbb{R}^{n}$, see [Bonnans and Shapiro, 2000, Section 2.1.4]. If $C_{1}, C_{2}$ are additionally, closed and convex, we have $\left(C_{1} \cap C_{2}\right)^{\circ}=\operatorname{cl}\left(C_{1}^{\circ}+C_{2}^{\circ}\right)$. Particularly, for subspaces $L_{1}, L_{2} \subset \mathbb{R}^{n}$, $\left(L_{1} \cap L_{2}\right)^{\perp}=L_{1}^{\perp}+L_{2}^{\perp}$ is valid since each subspace of $\mathbb{R}^{n}$ is closed. Supposing that $K \subset \mathbb{R}^{n}$ is a closed, convex cone, one obtains $K^{\circ \perp}=K \cap(-K)$ from the bipolar theorem, i.e. $K^{\circ \perp}$ coincides with the so-called lineality space of $K$ which is the largest subspace contained in $K$. Additionally,

$$
\begin{aligned}
K^{\perp \perp} & =\left(K^{\circ} \cap(-K)^{\circ}\right)^{\perp}=\left(K^{\circ} \cap(-K)^{\circ}\right)^{\circ} \\
& =\operatorname{cl}\left(K^{\circ \circ}+(-K)^{\circ \circ}\right)=\operatorname{cl}(K-K)=K-K=\operatorname{span} K
\end{aligned}
$$

follows from the calculation rules provided above.

\subsubsection{Tangent and normal cones}

Let $A \subset \mathbb{R}^{n}$ be closed and fix an arbitrary point $\bar{x} \in A$. The closed cones

$$
\begin{aligned}
& \mathcal{T}_{A}(\bar{x}):=\left\{d \in \mathbb{R}^{n} \mid \begin{array}{c}
\exists\left\{t_{k}\right\}_{k \in \mathbb{N}} \subset \mathbb{R}_{+} \exists\left\{d_{k}\right\}_{k \in \mathbb{N}} \subset \mathbb{R}^{n}: \\
t_{k} \downarrow 0, d_{k} \rightarrow d, \bar{x}+t_{k} d_{k} \in A \forall k \in \mathbb{N}
\end{array}\right\}, \\
& \mathcal{T}_{A}^{b}(\bar{x}):=\left\{d \in \mathbb{R}^{n} \mid \begin{array}{c}
\forall\left\{t_{k}\right\}_{k \in \mathbb{N}} \subset \mathbb{R}_{+}, t_{k} \downarrow 0 \\
\exists\left\{d_{k}\right\}_{k \in \mathbb{N}} \subset \mathbb{R}^{n}: d_{k} \rightarrow d, \bar{x}+t_{k} d_{k} \in A \forall k \in \mathbb{N}
\end{array}\right\}, \\
& \mathcal{T}_{A}^{\mathrm{c}}(\bar{x}):=\left\{d \in \mathbb{R}^{n} \mid \begin{array}{c}
\forall\left\{t_{k}\right\}_{k \in \mathbb{N}} \subset \mathbb{R}_{+}, t_{k} \downarrow 0 \forall\left\{x_{k}\right\}_{k \in \mathbb{N}} \subset A, x_{k} \rightarrow \bar{x} \\
\exists\left\{d_{k}\right\}_{k \in \mathbb{N}} \subset \mathbb{R}^{n}: d_{k} \rightarrow d, x_{k}+t_{k} d_{k} \in A \forall k \in \mathbb{N}
\end{array}\right\}
\end{aligned}
$$

are called the tangent (or Bouligand) cone, the inner (or adjacent) tangent cone, and the Clarke tangent cone to $A$ at $\bar{x}$, respectively. By definition, we always have the inclusions $\mathcal{T}_{A}^{\mathrm{c}}(\bar{x}) \subset \mathcal{T}_{A}^{\mathrm{b}}(\bar{x}) \subset \mathcal{T}_{A}(\bar{x})$, and all these cones coincide whenever $A$ is convex. If we have 
$\mathcal{T}_{A}^{b}(\bar{x})=\mathcal{T}_{A}(\bar{x})$, then $A$ is said to be derivable at $\bar{x}$. We say that $A$ is derivable if it is derivable at each of its points. The cone $\widehat{\mathcal{N}}_{A}(\bar{x}):=\mathcal{T}_{A}(\bar{x})^{\circ}$ is referred to as Fréchet (or regular) normal cone. By definition, it is closed and convex. Furthermore, we exploit the limiting (or Mordukhovich) normal cone to $A$ at $\bar{x}$ which is given by

$$
\mathcal{N}_{A}(\bar{x})=\left\{\eta \in \mathbb{R}^{n} \mid \begin{array}{c}
\exists\left\{x_{k}\right\}_{k \in \mathbb{N}} \subset A \exists\left\{\eta_{k}\right\}_{k \in \mathbb{N}} \subset \mathbb{R}^{n}: \\
x_{k} \rightarrow \bar{x}, \eta_{k} \rightarrow \eta, \eta_{k} \in \widehat{\mathcal{N}}_{A}\left(x_{k}\right) \forall k \in \mathbb{N}
\end{array}\right\} .
$$

Using the notion of the Painlevé-Kuratowski-limit, see e.g. [Rockafellar and Wets, 1998, Section 4.B], we have

$$
\mathcal{N}_{A}(\bar{x})=\limsup _{x \rightarrow \bar{x}, x \in A} \widehat{\mathcal{N}}_{A}(x) .
$$

Clearly, $\widehat{\mathcal{N}}_{A}(\bar{x}) \subset \mathcal{N}_{A}(\bar{x})$ holds and both cones coincide whenever $A$ is convex. In case where $A$ is a closed, convex cone, we obtain $\widehat{\mathcal{N}}_{A}(\bar{x})=A^{\circ} \cap\{\bar{x}\}^{\perp}$. For formal completeness, we set $\mathcal{T}_{A}(x)=\mathcal{T}_{A}^{b}(x)=\mathcal{T}_{A}^{\mathrm{c}}(x):=\varnothing$ and $\widehat{\mathcal{N}}_{A}(x)=\mathcal{N}_{A}(x):=\varnothing$ for each $x \notin A$.

Lemma 2.1. Let $Q \subset \mathbb{R}^{n}$ be a polyhedral set and fix $\bar{x} \in Q$. Then, there is some $\varepsilon>0$ such that we have

$$
\forall x \in Q \cap \mathbb{U}^{\varepsilon}(\bar{x}): \quad \widehat{\mathcal{N}}_{Q}(x)=\widehat{\mathcal{N}}_{Q}(\bar{x}) \cap\{x-\bar{x}\}^{\perp} .
$$

Proof. Since $Q$ is polyhedral, [Rockafellar and Wets, 1998, Exercise 6.47] yields the existence of $\varepsilon>0$ such that $Q \cap \mathbb{U}^{\varepsilon}(\bar{x})=\left(\{\bar{x}\}+\mathcal{T}_{Q}(\bar{x})\right) \cap \mathbb{U}^{\varepsilon}(\bar{x})$ is valid. Now, fix an arbitrary point $x \in Q \cap \mathbb{U}^{\varepsilon}(\bar{x})$. Noting that $x$ is an interior point of $\mathbb{U}^{\varepsilon}(\bar{x})$, we obtain

$$
\begin{aligned}
\widehat{\mathcal{N}}_{Q}(x) & =\widehat{\mathcal{N}}_{Q \cap \mathbb{U}^{\varepsilon}(\bar{x})}(x)=\widehat{\mathcal{N}}_{\left(\{\bar{x}\}+\mathcal{T}_{Q}(\bar{x})\right) \cap \mathbb{U}^{\varepsilon}(\bar{x})}(x)=\widehat{\mathcal{N}}_{\{\bar{x}\}+\mathcal{T}_{Q}(\bar{x})}(x) \\
& =\widehat{\mathcal{N}}_{\mathcal{T}_{Q}(\bar{x})}(x-\bar{x})=\mathcal{T}_{Q}(\bar{x})^{\circ} \cap\{x-\bar{x}\}^{\perp}=\widehat{\mathcal{N}}_{Q}(\bar{x}) \cap\{x-\bar{x}\}^{\perp}
\end{aligned}
$$

from the fact that $\mathcal{T}_{Q}(\bar{x})$ is a closed, convex cone. This completes the proof.

Lemma 2.2. Let $A:=\bigcup_{i=1}^{r} A_{i}$ be the finite union of closed sets $A_{1}, \ldots, A_{r} \subset \mathbb{R}^{n}$, choose $\bar{x} \in A$, and set $I(\bar{x}):=\left\{i \in\{1, \ldots, r\} \mid \bar{x} \in A_{i}\right\}$. Then, one has

$$
\begin{aligned}
& \mathcal{T}_{A}(\bar{x})=\bigcup_{i \in I(\bar{x})} \mathcal{T}_{A_{i}}(\bar{x}), \quad \mathcal{T}_{A}^{b}(\bar{x}) \supset \bigcup_{i \in I(\bar{x})} \mathcal{T}_{A_{i}}^{b}(\bar{x}), \quad \mathcal{T}_{A}^{\mathrm{c}}(\bar{x}) \supset \bigcap_{i \in I(\bar{x})} \mathcal{T}_{A_{i}}^{\mathrm{c}}(\bar{x}), \\
& \widehat{\mathcal{N}}_{A}(\bar{x})=\bigcap_{i \in I(\bar{x})} \widehat{\mathcal{N}}_{A_{i}}(\bar{x}), \quad \mathcal{N}_{A}(\bar{x}) \subset \bigcup_{i \in I(\bar{x})} \mathcal{N}_{A_{i}}(\bar{x})
\end{aligned}
$$

If, additionally, the sets $A_{1}, \ldots, A_{r}$ are convex, then $A$ is derivable. Furthermore, we particularly have

$$
\mathcal{N}_{A}(\bar{x}) \subset \bigcup_{i \in I(\bar{x})} \widehat{\mathcal{N}}_{A_{i}}(\bar{x})
$$

Proof. The formulas for the tangent and the inner tangent cone can be found in [Aubin and Frankowska, 2009, Tables 4.1 and 4.2]. Furthermore, the identity for the Fréchet normal cone follows 
from the formula for the tangent cone by polarization. The inclusion for the Clarke tangent cone follows by definition of this cone while observing that $A$ is the union of only finitely many sets. For the proof of the inclusion involving the limiting normal cone, observe that due to the closedness of all the sets $A_{1}, \ldots, A_{r}$, there is some ball $\mathbb{B}^{\varepsilon}(\bar{x})$ such that $I(x) \subset I(\bar{x})$ holds for all $x \in A \cap \mathbb{B}^{\varepsilon}(\bar{x})$. This yields

$$
\begin{aligned}
\mathcal{N}_{A}(\bar{x}) & =\limsup _{x \rightarrow \bar{x}, x \in A} \widehat{\mathcal{N}}_{A}(x)=\limsup _{x \rightarrow \bar{x}, x \in A} \bigcap_{i \in I(x)} \widehat{\mathcal{N}}_{A_{i}}(x) \\
& \subset \limsup _{x \rightarrow \bar{x}, x \in A} \bigcup_{i \in I(x)} \widehat{\mathcal{N}}_{A_{i}}(x) \subset \limsup _{x \rightarrow \bar{x}, x \in A} \bigcup_{i \in I(\bar{x})} \widehat{\mathcal{N}}_{A_{i}}(x) \\
& =\bigcup_{i \in I(\bar{x})} \limsup _{x \rightarrow \bar{x}, x \in A_{i}} \widehat{\mathcal{N}}_{A_{i}}(x)=\bigcup_{i \in I(\bar{x})} \mathcal{N}_{A_{i}}(\bar{x}) .
\end{aligned}
$$

Now, assume that $A_{1}, \ldots, A_{r}$ are convex. From above, we obtain

$$
\mathcal{T}_{A}(\bar{x})=\bigcup_{i \in I(\bar{x})} \mathcal{T}_{A_{i}}(\bar{x})=\bigcup_{i \in I(\bar{x})} \mathcal{T}_{A_{i}}^{b}(\bar{x}) \subset \mathcal{T}_{A}^{b}(\bar{x}) \subset \mathcal{T}_{A}(\bar{x})
$$

for each $\bar{x} \in A$ since each convex set is derivable. Thus, $A$ is derivable. Taking the above upper estimate for the limiting normal cone in mind, the final formula of the lemma follows by convexity of $A_{1}, \ldots, A_{r}$.

Lemma 2.3. Let $S:=\bigcup_{i=1}^{r} S_{i}$ be the finite union of polyhedral sets $S_{1}, \ldots, S_{r} \subset \mathbb{R}^{n}$. Fix a sequence $\left\{x_{k}\right\}_{k \in \mathbb{N}} \subset S$ converging to $\bar{x} \in \mathbb{R}^{n}$. For each $k \in \mathbb{N}$, let $\lambda_{k} \in \widehat{\mathcal{N}}_{S}\left(x_{k}\right)$ be chosen such that $\lambda_{k} \rightarrow \bar{\lambda}$ holds true for some $\bar{\lambda} \in \mathbb{R}^{n}$. Then, we have $\lambda_{k} \cdot\left(x_{k}-\bar{x}\right)=\bar{\lambda} \cdot\left(x_{k}-\bar{x}\right)=0$ for all sufficiently large $k \in \mathbb{N}$.

Proof. Let us set $I(x):=\left\{i \in\{1, \ldots, r\} \mid x \in S_{i}\right\}$ for each $x \in S$, see Lemma 2.2. Due to the closedness of $S, \bar{x} \in S$ is valid. Exploiting the closedness of $S_{1}, \ldots, S_{r}$ as well as the convergence $x_{k} \rightarrow \bar{x}$, the inclusion $I\left(x_{k}\right) \subset I(\bar{x})$ needs to be valid for all sufficiently large $k \in \mathbb{N}$. Particularly, for each large enough $k_{0} \in \mathbb{N}$, there is an index $i\left(k_{0}\right) \in I(\bar{x})$ such that $x_{k_{0}} \in S_{i\left(k_{0}\right)}$ holds. Now, we can exploit Lemma 2.1 in order to see the relation $\lambda_{k_{0}} \in \widehat{\mathcal{N}}_{S_{i\left(k_{0}\right)}}\left(x_{k_{0}}\right)=\widehat{\mathcal{N}}_{S_{i\left(k_{0}\right)}}(\bar{x}) \cap\left\{x_{k_{0}}-\bar{x}\right\}^{\perp}$ for large enough $k_{0} \in \mathbb{N}$, i.e. $\lambda_{k_{0}} \cdot\left(x_{k_{0}}-\bar{x}\right)=0$ follows.

Noting that $S$ is a finite union, for sufficiently large $k_{0} \in \mathbb{N}$, there is a subsequence $\left\{x_{k_{l}}\right\}_{l \in \mathbb{N}}$ of $\left\{x_{k}\right\}_{k \in \mathbb{N}}$ with $k_{l} \geq k_{0}$ for all $l \in \mathbb{N}$ and some index $i\left(k_{0}\right) \in I\left(x_{k_{0}}\right)$ such that $i\left(k_{0}\right) \in I\left(x_{k_{l}}\right) \subset I(\bar{x})$ is valid for all $l \in \mathbb{N}$. For large enough $l \in \mathbb{N}$, we particularly obtain $\lambda_{k_{l}} \in \widehat{\mathcal{N}}_{S_{i\left(k_{0}\right)}}\left(x_{k_{l}}\right)$ from Lemma 2.2. Noting that there exist only finitely many different Fréchet normal cones to a polyhedral set, we find a subsequence $\left\{x_{k_{l_{\nu}}}\right\}_{\nu \in \mathbb{N}}$ of $\left\{x_{k_{l}}\right\}_{l \in \mathbb{N}}$ and a polyhedral cone $K \subset \mathbb{R}^{n}$ such that $K=\widehat{\mathcal{N}}_{S_{i\left(k_{0}\right)}}\left(x_{k_{l_{\nu}}}\right)$ holds for all $\nu \in \mathbb{N}$, and for large enough $k_{0} \in \mathbb{N}$, we can even guarantee $K=\widehat{\mathcal{N}}_{S_{i\left(k_{0}\right)}}\left(x_{k_{0}}\right)$. Particularly, $\lambda_{k_{l_{\nu}}} \in K$ follows for all $\nu \in \mathbb{N}$. Noting that $\lambda_{k_{l_{\nu}}} \rightarrow \bar{\lambda}$ holds as $\nu \rightarrow \infty$, we have $\bar{\lambda} \in K$ by closedness of $K$. Finally, we observe that $K=\widehat{\mathcal{N}}_{S_{i\left(k_{0}\right)}}\left(x_{k_{0}}\right)=\widehat{\mathcal{N}}_{S_{i_{k_{0}}}}(\bar{x}) \cap\left\{x_{k_{0}}-\bar{x}\right\}^{\perp}$ holds due to Lemma 2.1. This shows $\bar{\lambda} \cdot\left(x_{k_{0}}-\bar{x}\right)=0$ for large enough $k_{0} \in \mathbb{N}$. 


\subsubsection{Second-order tangent sets}

For the consideration of second-order optimality conditions, we exploit so-called secondorder tangent sets. Therefore, let $A \subset \mathbb{R}^{n}$ be a closed set and fix $\bar{x} \in A$ as well as $d \in \mathcal{T}_{A}(\bar{x})$. The closed sets

$$
\begin{aligned}
& \mathcal{T}_{A}^{2}(\bar{x} ; d):=\left\{h \in \mathbb{R}^{n} \begin{array}{c}
\exists\left\{t_{k}\right\}_{k \in \mathbb{N}} \subset \mathbb{R}_{+} \exists\left\{h_{k}\right\}_{k \in \mathbb{N}} \subset \mathbb{R}^{n}: \\
t_{k} \downarrow 0, h_{k} \rightarrow h, \bar{x}+t_{k} d+\frac{1}{2} t_{k}^{2} h_{k} \in A \forall k \in \mathbb{N}
\end{array}\right\}, \\
& \mathcal{T}_{A}^{b, 2}(\bar{x} ; d):=\left\{h \in \mathbb{R}^{n} \mid \begin{array}{c}
\forall\left\{t_{k}\right\}_{k \in \mathbb{N}} \subset \mathbb{R}_{+}, t_{k} \downarrow 0 \\
\exists\left\{h_{k}\right\}_{k \in \mathbb{N}} \subset \mathbb{R}^{n}: h_{k} \rightarrow h, \bar{x}+t_{k} d+\frac{1}{2} t_{k}^{2} h_{k} \in A \forall k \in \mathbb{N}
\end{array}\right\}
\end{aligned}
$$

are called outer (Bouligand) and inner (adjacent) second-order tangent set to $A$ at $\bar{x}$ in direction $d$, see e.g. [Bonnans and Shapiro, 2000, Definition 3.28]. Note that these sets are not conic in general. For $\tilde{d} \notin \mathcal{T}_{A}(\bar{x})$, we set $\mathcal{T}_{A}^{2}(\bar{x} ; \tilde{d})=\mathcal{T}_{A}^{b, 2}(\bar{x}, \tilde{d}):=\varnothing$ for formal completeness. Clearly, we always have $\mathcal{T}_{A}^{b, 2}(\bar{x} ; d) \subset \mathcal{T}_{A}^{2}(\bar{x} ; d)$. If equality holds, then $A$ is called parabolically derivable at $\bar{x}$ in direction $d$. We say that $A$ is parabolically derivable if it is parabolically derivable at each point $x \in A$ in each direction $d \in \mathcal{T}_{A}(x)$. Note that even convex sets are not parabolically derivable in general. However, it follows from [Bonnans and Shapiro, 2000, Proposition 3.34] that each polyhedral set $Q \subset \mathbb{R}^{n}$ is parabolically derivable and it holds

$$
\forall x \in Q \forall d \in \mathcal{T}_{Q}(x): \quad \mathcal{T}_{Q}^{2}(x ; d)=\mathcal{T}_{Q}^{b, 2}(x ; d)=\mathcal{T}_{\mathcal{T}_{Q}(x)}(d),
$$

see [Rockafellar and Wets, 1998, Proposition 13.12] as well. From Lemma 2.2, we know that the union of finitely many polyhedral sets is derivable. In the subsequent lemma, we extend this result to parabolic derivability.

Lemma 2.4. Let $S_{1}, \ldots, S_{r} \subset \mathbb{R}^{n}$ be polyhedral sets and define $S:=\bigcup_{i=1}^{r} S_{i}$ Then, for each $x \in S$ and $d \in \mathcal{T}_{S}(x)$, we have

$$
\mathcal{T}_{S}^{2}(x ; d)=\mathcal{T}_{S}^{b, 2}(x ; d)=\mathcal{T}_{\mathcal{T}_{S}(x)}(d)
$$

and

$$
\mathcal{T}_{S}^{2}(x ; d)+\bigcap_{i \in I(x)} \mathcal{T}_{S_{i}}(x)^{\circ \perp} \subset \mathcal{T}_{S}^{2}(x ; d)
$$

where we used $I(x):=\left\{i \in\{1, \ldots, r\} \mid x \in S_{i}\right\}$. In particular, $S$ is parabolically derivable.

Proof. Fix $x \in S$ and $d \in \mathcal{T}_{S}(x)$. We exploit the calculus rules from [Bonnans and Shapiro, 2000, Proposition 3.37] and the fact that polyhedral sets are parabolically derivable in order to obtain

$$
\mathcal{T}_{S}^{2}(x ; d)=\bigcup_{i \in I(x)} \mathcal{T}_{S_{i}}^{2}(x ; d)=\bigcup_{i \in I(x)} \mathcal{T}_{S_{i}}^{b, 2}(x ; d) \subset \mathcal{T}_{S}^{b, 2}(x ; d) \subset \mathcal{T}_{S}^{2}(x ; d)
$$

This already shows the parabolic derivability of $S$ at $x$ in direction $d$. 
Next, we use formula (1) as well as Lemma 2.2 in order to see

$$
\mathcal{T}_{S}^{2}(x ; d)=\bigcup_{i \in I(x)} \mathcal{T}_{S_{i}}^{2}(x ; d)=\bigcup_{i \in I(x)} \mathcal{T}_{\mathcal{T}_{S_{i}}(x)}(d)=\mathcal{T}_{\bigcup_{i \in I(x)}} \mathcal{T}_{S_{i}}(x)(d)=\mathcal{T}_{\mathcal{T}_{S}(x)}(d) .
$$

In order to prove correctness of the last formula, we first invoke [Rockafellar and Wets, 1998, Proposition 13.12] in order to see that

$$
\mathcal{T}_{S_{i}}^{2}(x ; d)+\mathcal{T}_{S_{i}}(x) \subset \mathcal{T}_{S_{i}}^{2}(x ; d)
$$

holds true for all $i \in I(x)$ since $S_{i}$ is a polyhedron. This leads to

$$
\begin{aligned}
\mathcal{T}_{S}^{2}(x ; d) & +\bigcap_{i \in I(x)} \mathcal{T}_{S_{i}}(x)^{\circ \perp}=\left(\bigcup_{j \in I(x)} \mathcal{T}_{S_{j}}^{2}(x ; d)\right)+\bigcap_{i \in I(x)} \mathcal{T}_{S_{i}}(x)^{\circ \perp} \\
& =\bigcup_{j \in I(x)}\left(\mathcal{T}_{S_{j}}^{2}(x ; d)+\bigcap_{i \in I(x)} \mathcal{T}_{S_{i}}(x)^{\circ \perp}\right) \subset \bigcup_{j \in I(x)}\left(\mathcal{T}_{S_{j}}^{2}(x ; d)+\mathcal{T}_{S_{j}}(x)^{\circ \perp}\right) \\
& \subset \bigcup_{j \in I(x)}\left(\mathcal{T}_{S_{j}}^{2}(x ; d)+\mathcal{T}_{S_{j}}(x)\right) \subset \bigcup_{j \in I(x)} \mathcal{T}_{S_{j}}^{2}(x ; d)=\mathcal{T}_{S}^{2}(x ; d)
\end{aligned}
$$

and completes the proof.

\subsubsection{Inverse images}

Next, we present some preliminary results on the variational geometry associated with preimages of closed sets under smooth transformations. Therefore, let $P: \mathbb{R}^{n} \rightarrow \mathbb{R}^{m}$ be a twice continuously differentiable mapping and let $\Omega \subset \mathbb{R}^{m}$ be a closed set such that $Y:=\left\{x \in \mathbb{R}^{n} \mid P(x) \in \Omega\right\}$ is nonempty. For our subsequent considerations, we fix $\bar{x} \in Y$.

Let us first discuss variational approximations of tangents to $Y$ at $\bar{x}$. We call

$$
\mathcal{L}_{Y}(\bar{x}):=\left\{d \in \mathbb{R}^{n} \mid \nabla P(\bar{x}) d \in \mathcal{T}_{\Omega}(P(\bar{x}))\right\}
$$

the linearization cone to $Y$ at $\bar{x}$. One always has the inclusion $\mathcal{T}_{Y}(\bar{x}) \subset \mathcal{L}_{Y}(\bar{x})$, see [Rockafellar and Wets, 1998, Theorem 6.31], while equality holds if the so-called feasibility mapping $\mathbb{R}^{n} \ni x \mapsto\{P(x)\}-\Omega \subset \mathbb{R}^{m}$ is metrically subregular at $\left(\bar{x}, 0^{m}\right)$, see [Henrion and Outrata, 2005, Proposition 1]. The latter condition has been named metric subregularity constraint qualification (MSCQ) in [Gfrerer and Mordukhovich, 2015, Definition 3.2]. As it is mentioned in Henrion and Outrata [2005], the validity of the so-called no nonzero abnormal multiplier constraint qualification (NNAMCQ) given by

$$
0^{n}=\nabla P(\bar{x})^{\top} \lambda, \lambda \in \mathcal{N}_{\Omega}(P(\bar{x})) \Longrightarrow \lambda=0^{m}
$$

is sufficient for MSCQ to hold. In the literature, NNAMCQ is sometimes called generalized Mangasarian-Fromovitz constraint qualification (GMFCQ) since it reduces to the classical MFCQ condition in the context of standard nonlinear programming. Below, we show that MSCQ can be used in order to obtain a precise characterization of the outer second-order tangent set associated with $Y$. For the proof, we follow ideas from [Rockafellar and Wets, 1998, Proposition 13.13]. 
Lemma 2.5. Let $\bar{x} \in Y$ be arbitrarily chosen. Then, for each $d \in \mathcal{T}_{Y}(\bar{x})$, we have

$$
\mathcal{T}_{Y}^{2}(\bar{x} ; d) \subset\left\{h \in \mathbb{R}^{n} \mid \nabla P(\bar{x}) h+\nabla^{2} P(\bar{x})[d, d] \in \mathcal{T}_{\Omega}^{2}(P(\bar{x}) ; \nabla P(\bar{x}) d)\right\} .
$$

If $M S C Q$ is valid at $\bar{x}$, then equality holds.

Proof. We start proving the inclusion $\subset$ which is supposed to be valid in general. Fix $h \in \mathcal{T}_{Y}^{2}(\bar{x} ; d)$ arbitrarily. Then, we find sequences $\left\{h_{k}\right\}_{k \in \mathbb{N}} \subset \mathbb{R}^{n}$ and $\left\{t_{k}\right\}_{k \in \mathbb{N}} \subset \mathbb{R}_{+}$such that $h_{k} \rightarrow h, t_{k} \downarrow 0$, as well as $P\left(\bar{x}+t_{k} d+\frac{1}{2} t_{k}^{2} h_{k}\right) \in \Omega$ for all $k \in \mathbb{N}$ hold true. For each $k \in \mathbb{N}$, we now define

$$
r_{k}:=\frac{P\left(\bar{x}+t_{k} d+\frac{1}{2} t_{k}^{2} h_{k}\right)-P(\bar{x})-t_{k} \nabla P(\bar{x}) d}{\frac{1}{2} t_{k}^{2}} .
$$

Then, we have $P(\bar{x})+t_{k} \nabla P(\bar{x}) d+\frac{1}{2} t_{k}^{2} r_{k}=P\left(\bar{x}+t_{k} d+\frac{1}{2} t_{k}^{2} h_{k}\right) \in \Omega$ for all $k \in \mathbb{N}$, i.e. supposing that $\left\{r_{k}\right\}_{k \in \mathbb{N}}$ converges, its limit belongs to $\mathcal{T}_{\Omega}^{2}(P(\bar{x}) ; \nabla P(\bar{x}) d)$. On the other hand, we have

$$
r_{k}=\frac{P\left(\bar{x}+t_{k}\left(d+\frac{1}{2} t_{k} h_{k}\right)\right)-P(\bar{x})-t_{k} \nabla P(\bar{x})\left(d+\frac{1}{2} t_{k} h_{k}\right)}{\frac{1}{2} t_{k}^{2}}+\nabla P(\bar{x}) h_{k}
$$

and this sum converges to $\nabla^{2} P(\bar{x})[d, d]+\nabla P(\bar{x}) h$, see e.g. [Rockafellar and Wets, 1998, Example 13.8]. Thus, we have $\nabla^{2} P(\bar{x})[d, d]+\nabla P(\bar{x}) h \in \mathcal{T}_{\Omega}^{2}(P(\bar{x}) ; \nabla P(\bar{x}) d)$.

Now, we assume that MSCQ holds at $\bar{x}$ and show validity of the converse inclusion $\supset$. Fix $h \in \mathbb{R}^{n}$ satisfying $\nabla P(\bar{x}) h+\nabla^{2} P(\bar{x})[d, d] \in \mathcal{T}_{\Omega}^{2}(P(\bar{x}) ; \nabla P(\bar{x}) d)$. Then, we find sequences $\left\{r_{k}\right\}_{k \in \mathbb{N}} \subset \mathbb{R}^{m}$ and $\left\{t_{k}\right\}_{k \in \mathbb{N}} \subset \mathbb{R}_{+}$such that $r_{k} \rightarrow \nabla P(\bar{x}) h+\nabla^{2} P(\bar{x})[d, d]$, $t_{k} \downarrow 0$, and $P(\bar{x})+t_{k} \nabla P(\bar{x}) d+\frac{1}{2} t_{k}^{2} r_{k} \in \Omega$ for all $k \in \mathbb{N}$. Noting that $Y$ is closed, let us fix $x_{k} \in \operatorname{argmin}\left\{\left\|\bar{x}+t_{k} d+\frac{1}{2} t_{k}^{2} h-x\right\|_{2} \mid x \in Y\right\}$ for all $k \in \mathbb{N}$. Since the feasibility map $\mathbb{R}^{n} \ni x \mapsto\{P(x)\}-\Omega \subset \mathbb{R}^{m}$ is metrically subregular at $\left(\bar{x}, 0^{m}\right)$, we find constants $\kappa>0$ and $\varepsilon>0$ such that

$$
\forall x \in \mathbb{U}^{\varepsilon}(\bar{x}): \quad \operatorname{dist}(x, Y) \leq \kappa \operatorname{dist}(P(x), \Omega) .
$$

We obtain

$$
\begin{gathered}
\left\|\frac{x_{k}-\bar{x}-t_{k} d}{\frac{1}{2} t_{k}^{2}}-h\right\|_{2}=\frac{2}{t_{k}^{2}} \operatorname{dist}\left(\bar{x}+t_{k} d+\frac{1}{2} t_{k}^{2} h, Y\right) \leq \frac{2 \kappa}{t_{k}^{2}} \operatorname{dist}\left(P\left(\bar{x}+t_{k} d+\frac{1}{2} t_{k}^{2} h\right), \Omega\right) \\
\leq \kappa\left\|\frac{P\left(\bar{x}+t_{k} d+\frac{1}{2} t_{k}^{2} h\right)-P(\bar{x})-t_{k} \nabla P(\bar{x}) d}{\frac{1}{2} t_{k}^{2}}-r_{k}\right\|_{2} \\
=\kappa\left\|\frac{P\left(\bar{x}+t_{k}\left(d+\frac{1}{2} t_{k} h\right)\right)-P(\bar{x})-t_{k} \nabla P(\bar{x})\left(d+\frac{1}{2} t_{k} h\right)}{\frac{1}{2} t_{k}^{2}}+\nabla P(\bar{x}) h-r_{k}\right\|_{2}
\end{gathered}
$$

for sufficiently large $k \in \mathbb{N}$ and the last term converges to 0 as $k \rightarrow \infty$, see [Rockafellar and Wets, 1998, Example 13.8]. Thus, we have shown $h \in \mathcal{T}_{Y}^{2}(\bar{x} ; d)$. 
Let us now focus on the variational description of Fréchet normals to $Y$ at $\bar{x}$. Similarly as above, we generally have

$$
\widehat{\mathcal{N}}_{Y}(\bar{x}) \supset \nabla P(\bar{x})^{\top} \widehat{\mathcal{N}}_{\Omega}(P(\bar{x}))
$$

while the converse inclusion can only be guaranteed postulating additional assumptions. The following result is associated with this issue and taken from [Gfrerer and Outrata, 2016, Theorem 4].

Proposition 2.6. Let $\bar{x} \in Y$ be a point where $M S C Q$ holds. Suppose that there exists a subspace $L \subset \mathbb{R}^{m}$ satisfying $\mathcal{T}_{\Omega}(P(\bar{x}))+L \subset \mathcal{T}_{\Omega}(P(\bar{x}))$ and

$$
\nabla P(\bar{x}) \mathbb{R}^{n}+L=\mathbb{R}^{m} .
$$

Then, equality holds in (3).

In the context of certain instances of disjunctive programming, there exist weaker conditions than those ones postulated in Proposition 2.6 which ensure equality in (3), see Benko and Gfrerer [2017].

\section{An MPDC-tailored version of the linear independence constraint qualification}

We start this section by defining the constraint qualification of our interest. Recall that $X \subset \mathbb{R}^{n}$ denotes the feasible set of (MPDC).

Definition 3.1. Let $\bar{x} \in X$ be an arbitrary feasible point of (MPDC). Then, the linear independence constraint qualification (MPDC-LICQ) is said to hold at $\bar{x}$ if the following condition is valid:

$$
0^{n}=\nabla F(\bar{x})^{\top} \lambda, \lambda \in \sum_{i \in I(\bar{x})} \operatorname{span} \widehat{\mathcal{N}}_{D_{i}}(F(\bar{x})) \Longrightarrow \lambda=0^{m} .
$$

Here, we used $I(\bar{x}):=\left\{i \in\{1, \ldots, r\} \mid F(\bar{x}) \in D_{i}\right\}$.

We first note that MPDC-LICQ holds at $\bar{x} \in X$ whenever the matrix $\nabla F(\bar{x})$ possesses full row rank $m$, i.e. if the gradients $\nabla F_{i}(\bar{x}), \ldots, \nabla F_{m}(\bar{x})$ of the component mappings $F_{1}, \ldots, F_{m}: \mathbb{R}^{n} \rightarrow \mathbb{R}$ associated with $F$ are linearly independent. In the example below, it will be demonstrated that MPDC-LICQ reduces to the well-known LICQ whenever standard nonlinear programs are under consideration.

Example 3.2. For continuously differentiable functions $g_{1}, \ldots, g_{p}, h_{1}, \ldots, h_{q}: \mathbb{R}^{n} \rightarrow \mathbb{R}$, we consider the standard nonlinear program

$$
\begin{aligned}
f(x) & \rightarrow \min & & \\
g_{j}(x) & \leq 0 & & j=1, \ldots, p \\
h_{j}(x) & =0 & & j=1, \ldots, q .
\end{aligned}
$$


In order to transfer it to a program of type (MPDC), we choose $r:=1$, set $D:=\mathbb{R}_{-}^{p} \times\left\{0^{q}\right\}$, and define $F: \mathbb{R}^{n} \rightarrow \mathbb{R}^{p+q}$ by means of

$$
\forall x \in \mathbb{R}^{n}: \quad F(x):=\left[g(x)^{\top} \quad h(x)^{\top}\right]^{\top} .
$$

Here, the mappings $g: \mathbb{R}^{n} \rightarrow \mathbb{R}^{p}$ and $h: \mathbb{R}^{n} \rightarrow \mathbb{R}^{q}$ possess the component mappings $g_{1}, \ldots, g_{p}$ and $h_{1}, \ldots, h_{q}$, respectively.

Fix a feasible point $\bar{x} \in X$ of (NLP) and define $I^{g}(\bar{x}):=\left\{j \in\{1, \ldots, p\} \mid g_{j}(\bar{x})=0\right\}$. Using the calculus rules for the tangent and Fréchet normal cone to Cartesian products of (convex) sets, see [Rockafellar and Wets, 1998, Proposition 6.41], we have

$$
\mathcal{T}_{D}(F(\bar{x}))=\mathcal{T}_{\mathbb{R}_{-}^{p}}(g(\bar{x})) \times \mathcal{T}_{\left\{0^{q}\right\}}(h(\bar{x})), \quad \widehat{\mathcal{N}}_{D}(F(\bar{x}))=\widehat{\mathcal{N}}_{\mathbb{R}_{-}^{p}}(g(\bar{x})) \times \widehat{\mathcal{N}}_{\left\{0^{q}\right\}}(h(\bar{x})) .
$$

Straightforward calculations lead to the formulas

$$
\begin{array}{rlrl}
\mathcal{T}_{\mathbb{R}_{-}^{p}}(g(\bar{x})) & =\left\{d \in \mathbb{R}^{p} \mid \forall j \in I^{g}(\bar{x}): d_{j} \leq 0\right\}, & & \mathcal{T}_{\left\{0^{q}\right\}}(h(\bar{x}))=\left\{0^{q}\right\}, \\
\widehat{\mathcal{N}}_{\mathbb{R}_{-}^{p}}(g(\bar{x}))=\left\{\lambda \in \mathbb{R}_{+}^{p} \mid \forall j \notin I^{g}(\bar{x}): \lambda_{j}=0\right\}, & & \widehat{\mathcal{N}}_{\left\{0^{q}\right\}}(h(\bar{x}))=\mathbb{R}^{q},
\end{array}
$$

i.e. we have

$$
\operatorname{span} \widehat{\mathcal{N}}_{D}(F(\bar{x}))=\left\{\lambda \in \mathbb{R}^{p} \mid \forall j \notin I^{g}(\bar{x}): \lambda_{j}=0\right\} \times \mathbb{R}^{q} .
$$

Thus, MPDC-LICQ from Definition 3.1 takes the following form for (NLP):

$$
\left.\begin{array}{l}
0^{n}=\nabla g(\bar{x})^{\top} \lambda+\nabla h(\bar{x})^{\top} \rho, \\
\forall j \notin I^{g}(\bar{x}): \lambda_{j}=0
\end{array}\right\} \Longrightarrow \lambda=0^{p}, \rho=0^{q} .
$$

This is equivalent to the linear independence of the vectors from

$$
\left\{\nabla g_{j}(\bar{x}) \mid j \in I^{g}(\bar{x})\right\} \cup\left\{\nabla h_{j}(\bar{x}) \mid j \in\{1, \ldots, q\}\right\}
$$

which is precisely the definition of the standard linear independence constraint qualification from nonlinear programming.

In Section 5, we will show that in the particular instances of MPCCs, MPVCs, CCMPs, and MPSCs, MPDC-LICQ coincides with the well-known respective problem-tailored version of LICQ.

We provide an equivalent primal characterization of MPDC-LICQ in the subsequent lemma.

Lemma 3.3. Fix $\bar{x} \in X$ arbitrarily. Then, $M P D C-L I C Q$ is valid at $\bar{x}$ if and only if the subsequent condition is satisfies:

$$
\nabla F(\bar{x}) \mathbb{R}^{n}+\bigcap_{i \in I(\bar{x})} \mathcal{T}_{D_{i}}(F(\bar{x}))^{\circ \perp}=\mathbb{R}^{m} .
$$


Proof. First, we note that for any matrix $A \in \mathbb{R}^{m \times n}$ and any subspace $L \subset \mathbb{R}^{m}$, the equivalence

$$
A \mathbb{R}^{n}+L=\mathbb{R}^{m} \Longleftrightarrow\left\{\lambda \in L^{\perp} \mid A^{\top} \lambda=0^{n}\right\}=\left\{0^{m}\right\}
$$

follows from the polarization rules provided in Section 2.2. Thus, the statement of the lemma follows from setting $A:=\nabla F(\bar{x})$ as well as $L:=\bigcap_{i \in I(\bar{x})} \mathcal{T}_{D_{i}}(F(\bar{x}))^{\circ \perp}$ and observing that

$$
\left(\bigcap_{i \in I(\bar{x})} \mathcal{T}_{D_{i}}(F(\bar{x}))^{\circ \perp}\right)^{\perp}=\sum_{i \in I(\bar{x})} \widehat{\mathcal{N}}_{D_{i}}(F(\bar{x}))^{\perp \perp}=\sum_{i \in I(\bar{x})} \operatorname{span} \widehat{\mathcal{N}}_{D_{i}}(F(\bar{x}))
$$

holds true.

Remark 3.4. Fix $\bar{x} \in X$ arbitrarily. Due to

$$
\mathcal{T}_{D}(F(\bar{x}))^{\circ \perp}=\left(\bigcap_{i \in I(\bar{x})} \mathcal{T}_{D_{i}}(F(\bar{x}))^{\circ}\right)^{\perp} \supset \sum_{i \in I(\bar{x})} \mathcal{T}_{D_{i}}(F(\bar{x}))^{\circ \perp} \supset \bigcap_{i \in I(\bar{x})} \mathcal{T}_{D_{i}}(F(\bar{x}))^{\circ \perp}
$$

and Lemma 3.3, the validity of MPDC-LICQ at $\bar{x}$ implies that

$$
\nabla F(\bar{x}) \mathbb{R}^{n}+\mathcal{T}_{D}(F(\bar{x}))^{\circ \perp}=\mathbb{R}^{m}
$$

holds. The latter condition is referred to as nondegeneracy in the setting where $D$ is convex, see [Bonnans and Shapiro, 2000, Section 6.4.1]. Noting that D is typically nonconvex in our setting, we would like to mention that a related conditions in the context of disjunctive programming can be found in [Gfrerer, 2014, Definition 3.6].

The following lemma will be important for our remaining considerations.

Lemma 3.5. For each feasible point $\bar{x} \in X$ of (MPDC), the following conditions hold:

$$
\begin{aligned}
& \mathcal{T}_{D}(F(\bar{x}))+\bigcap_{i \in I(\bar{x})} \mathcal{T}_{D_{i}}(F(\bar{x}))^{\circ \perp} \subset \mathcal{T}_{D}(F(\bar{x})), \\
& \mathcal{N}_{D}(F(\bar{x})) \subset \sum_{i \in I(\bar{x})} \operatorname{span} \widehat{\mathcal{N}}_{D_{i}}(F(\bar{x})) .
\end{aligned}
$$

Proof. Using Lemma 2.2 and the convexity of $D_{1}, \ldots, D_{r}$, we find

$$
\begin{aligned}
\mathcal{T}_{D}(F(\bar{x}))+\bigcap_{i \in I(\bar{x})} \mathcal{T}_{D_{i}}(F(\bar{x}))^{\circ \perp} & =\left(\bigcup_{j \in I(\bar{x})} \mathcal{T}_{D_{j}}(F(\bar{x}))\right)+\bigcap_{i \in I(\bar{x})} \mathcal{T}_{D_{i}}(F(\bar{x}))^{\circ \perp} \\
& =\bigcup_{j \in I(\bar{x})}\left(\mathcal{T}_{D_{j}}(F(\bar{x}))+\bigcap_{i \in I(\bar{x})} \mathcal{T}_{D_{i}}(F(\bar{x}))^{\circ \perp}\right) \\
& \subset \bigcup_{j \in I(\bar{x})}\left(\mathcal{T}_{D_{j}}(F(\bar{x}))+\mathcal{T}_{D_{j}}(F(\bar{x}))^{\circ \perp}\right)
\end{aligned}
$$




$$
\begin{aligned}
& =\bigcup_{j \in I(\bar{x})}\left(\mathcal{T}_{D_{j}}(F(\bar{x}))+\mathcal{T}_{D_{j}}(F(\bar{x})) \cap\left(-\mathcal{T}_{D_{j}}(F(\bar{x}))\right)\right) \\
& =\bigcup_{j \in I(\bar{x})} \mathcal{T}_{D_{j}}(F(\bar{x}))=\mathcal{T}_{D}(F(\bar{x}))
\end{aligned}
$$

since we have $C+C \cap(-C) \subset C+C=C \subset C+C \cap(-C)$ for any closed, convex cone $C \subset \mathbb{R}^{m}$. This shows the validity of (5a). For the proof of (5b), we invoke Lemma 2.2 in order to see

$$
\mathcal{N}_{D}(F(\bar{x})) \subset \bigcup_{i \in I(\bar{x})} \widehat{\mathcal{N}}_{D_{i}}(F(\bar{x})) \subset \sum_{i \in I(\bar{x})} \widehat{\mathcal{N}}_{D_{i}}(F(\bar{x})) \subset \sum_{i \in I(\bar{x})} \operatorname{span} \widehat{\mathcal{N}}_{D_{i}}(F(\bar{x})) .
$$

This already completes the proof.

We combine the above lemma with Proposition 2.6 and Lemma 3.3 in order to obtain the following result.

Corollary 3.6. Let $\bar{x} \in X$ be a feasible point of (MPDC) where MPDC-LICQ is valid. Then, NNAMCQ is valid for (MPDC) at $\bar{x}$. Furthermore, we have

$$
\widehat{\mathcal{N}}_{X}(\bar{x})=\nabla F(\bar{x})^{\top} \widehat{\mathcal{N}}_{D}(F(\bar{x})) .
$$

Proof. The validity of NNAMCQ for (MPDC) at $\bar{x}$ follows from (5b) and the definition of MPDC-LICQ. Particularly, MSCQ holds for (MPDC) at $\bar{x}$. Now, we can combine the observation with (5a), Proposition 2.6, and Lemma 3.3 in order to finish the proof.

Clearly, our definition of MPDC-LICQ from Definition 3.1 is motivated by Proposition 2.6. Thus, the main issue here is the choice of a reasonable subspace $L \subset \mathbb{R}^{m}$ such that the condition

$$
\mathcal{T}_{D}(F(\bar{x}))+L \subset \mathcal{T}_{D}(F(\bar{x}))
$$

holds for a fixed feasible point $\bar{x} \in X$ of (MPDC). As we have seen in Lemma 3.5, the subspace $\bigcap_{i \in I(\bar{x})} \mathcal{T}_{D_{i}}(F(\bar{x}))^{\circ \perp}$ satisfies this condition while its annihilator is an upper approximation of $\mathcal{N}_{D}(F(\bar{x}))$. We note that the validity of (6) already implies the relation $\mathcal{T}_{D}(F(\bar{x})) \cup L \subset \mathcal{T}_{D}(F(\bar{x}))$ which yields $\widehat{\mathcal{N}}_{D}(F(\bar{x})) \cap L^{\perp} \supset \widehat{\mathcal{N}}_{D}(F(\bar{x}))$ by polarization and, thus, $L^{\perp} \supset \widehat{\mathcal{N}}_{D}(F(\bar{x}))$. Consequently, Lemma 2.2 shows that $L$ necessarily needs to satisfy $L \subset\left(\bigcap_{i \in I(\bar{x})} \mathcal{T}_{D_{i}}(F(\bar{x}))^{\circ}\right)^{\perp}$. Due to (4), another reasonable candidate for the choice of $L$ would be $\sum_{i \in I(\bar{x})} \mathcal{T}_{D_{i}}(F(\bar{x}))^{\circ \perp}$. However, considering e.g. $r=m:=2$, $F(\bar{x}):=0^{2}, D_{1}:=\mathbb{R} \times\{0\}$, and $D_{2}:=\{0\} \times \mathbb{R}^{+}$, one can easily check that this subspace is still too large since it violates the condition (6). Nevertheless, it might be possible that there is a subspace $L$ satisfying

$$
\bigcap_{i \in I(\bar{x})} \mathcal{T}_{D_{i}}(F(\bar{x}))^{\circ \perp} \subsetneq L \subsetneq \sum_{i \in I(\bar{x})} \mathcal{T}_{D_{i}}(F(\bar{x}))^{\circ \perp}
$$

as well as (6). This way, the resulting LICQ-type condition $\nabla F(\bar{x}) \mathbb{R}^{n}+L=\mathbb{R}^{m}$ would be less restrictive than MPDC-LICQ from Definition 3.1. However, it is not clear whether 
this condition can be used to infer all the results of this paper which are mainly valid under MPDC-LICQ.

Let us briefly interrelate the constraint qualification MPDC-LICQ with other prominent constraint qualifications from disjunctive programming.

Remark 3.7. Let $\bar{x} \in X$ be a feasible point of (MPDC) where MPDC-LICQ is valid. Then, due to Corollary 3.6, we obtain that the constraint qualifications NNAMCQ and $M S C Q$ hold for (MPDC) at $\bar{x}$ as well. Particularly, we obtain $\mathcal{T}_{X}(\bar{x})=\mathcal{L}_{X}(\bar{x})$ where $\mathcal{L}_{X}(\bar{x})$ denotes the linearization cone to $X$ at $\bar{x}$, see (2). In the literature of disjunctive programming, this condition is called generalized Abadie constraint qualification (GACQ), see [Flegel et al., 2007, Definition 6]. Furthermore, we obtain $\widehat{\mathcal{N}}_{X}(\bar{x})=\mathcal{L}_{X}(\bar{x})^{\circ}$ by polarization, and the latter condition is called generalized Guignard constraint qualification (GGCQ), see [Flegel et al., 2007, Definition 6].

Now, it is possible to exploit Proposition 2.6 in order to derive necessary optimality conditions of strong stationarity-type for (MPDC).

Theorem 3.8. Let $\bar{x} \in \mathbb{R}^{n}$ be a locally optimal solution of (MPDC) where MPDC-LICQ is valid. Then, there exists a uniquely determined multiplier $\lambda \in \mathbb{R}^{m}$ such that we have

$$
0^{n}=\nabla f(\bar{x})+\nabla F(\bar{x})^{\top} \lambda, \lambda \in \bigcap_{i \in I(\bar{x})} \widehat{\mathcal{N}}_{D_{i}}(F(\bar{x})) .
$$

Proof. Due to [Rockafellar and Wets, 1998, Theorem 6.12], we have $-\nabla f(\bar{x}) \in \widehat{\mathcal{N}}_{X}(\bar{x})$. Invoking Lemma 2.2 and Corollary 3.6, we obtain

$$
\widehat{\mathcal{N}}_{X}(\bar{x})=\nabla F(\bar{x})^{\top} \widehat{\mathcal{N}}_{D}(F(\bar{x}))=\nabla F(\bar{x})^{\top}\left[\bigcap_{i \in I(\bar{x})} \widehat{\mathcal{N}}_{D_{i}}(F(\bar{x}))\right],
$$

i.e. the postulated stationarity system possesses a solution.

It remains to show that the associated multiplier is uniquely determined. Therefore, assume that there are $\lambda^{1}, \lambda^{2} \in \bigcap_{i \in I(\bar{x})} \widehat{\mathcal{N}}_{D_{i}}(F(\bar{x}))$ satisfying $0^{n}=\nabla f(\bar{x})+\nabla F(\bar{x})^{\top} \lambda^{s}$, $s=1,2$. This yields $0^{n}=\nabla F(\bar{x})^{\top}\left(\lambda^{1}-\lambda^{2}\right)$. Moreover, for each $i \in I(\bar{x})$, we have

$$
\lambda^{1}-\lambda^{2} \in \widehat{\mathcal{N}}_{D_{i}}(F(\bar{x}))-\widehat{\mathcal{N}}_{D_{i}}(F(\bar{x}))=\operatorname{span} \widehat{\mathcal{N}}_{D_{i}}(F(\bar{x})) .
$$

This yields $\lambda^{1}-\lambda^{2} \in \sum_{i \in I(\bar{x})} \operatorname{span} \widehat{\mathcal{N}}_{D_{i}}(F(\bar{x}))$, and by validity of MPDC-LICQ, $\lambda^{1}=\lambda^{2}$ follows. This completes the proof.

Note that the multiplier $\lambda$ in Theorem 3.8 is chosen from the Fréchet normal cone $\widehat{\mathcal{N}}_{D}(F(\bar{x}))$. Keeping [Flegel et al., 2007, Definition 1] in mind, this observation justifies to call the above necessary optimality condition a strong stationarity-type condition.

Definition 3.9. A feasible point $\bar{x} \in X$ of (MPDC) is called strongly stationary ( $S$ stationary for short) if any only if there exists a multiplier $\lambda \in \bigcap_{i \in I(\bar{x})} \widehat{\mathcal{N}}_{D_{i}}(F(\bar{x}))$ which satisfies $0^{n}=\nabla f(\bar{x})+\nabla F(\bar{x})^{\top} \lambda$. 
Some general considerations regarding S-stationary points of disjunctive programs can be found in Flegel et al. [2007], Benko and Gfrerer [2017, 2018]. We note that for prominent classes of disjunctive programs like MPCCs, MPVCs, CCMPs, and MPSCs, there exist respective strong stationarity notions which can be obtained by applying Definition 3.9 to the specific problem setting, see Section 5. With the aid of Example 3.2, it is easily seen that for (NLP), the S-stationarity system equals the classical KarushKuhn-Tucker conditions.

Due to Remark 3.7, the validity of MPDC-LICQ at $\bar{x}$ implies that the tangent cone to $X$ at $\bar{x}$ equals the associated linearization cone. As we will see in the lemmas below, we also obtain derivability of $X$ at $\bar{x}$ as well as a nice representation of the second-order tangent sets to $X$ at $\bar{x}$ in each direction $d \in \mathcal{T}_{X}(\bar{x})$.

Lemma 3.10. Let $\bar{x} \in X$ be a feasible point of (MPDC) where MPDC-LICQ is valid. Then, $X$ is derivable at $\bar{x}$.

Proof. Due to Lemma 2.2, we obtain the inclusions

$$
\bigcap_{i \in I(\bar{x})} \mathcal{T}_{D_{i}}(F(\bar{x}))^{\circ \perp} \subset \bigcap_{i \in I(\bar{x})} \mathcal{T}_{D_{i}}(F(\bar{x}))=\bigcap_{i \in I(\bar{x})} \mathcal{T}_{D_{i}}^{\mathrm{c}}(F(\bar{x})) \subset \mathcal{T}_{D}^{\mathrm{c}}(F(\bar{x})) .
$$

Invoking Lemma 3.3, the validity of MPDC-LICQ yields

$$
\nabla F(\bar{x}) \mathbb{R}^{n}+\mathcal{T}_{D}^{\mathrm{c}}(F(\bar{x}))=\mathbb{R}^{m} .
$$

Thus, [Aubin and Frankowska, 2009, Theorem 4.3.3] can be applied in order to obtain

$$
\mathcal{T}_{X}^{b}(\bar{x})=\left\{d \in \mathbb{R}^{n} \mid \nabla F(\bar{x}) d \in \mathcal{T}_{D}^{b}(F(\bar{x}))\right\} .
$$

Since $D$ is derivable at $\bar{x}$, see Lemma 2.2, this yields $\mathcal{T}_{X}^{b}(\bar{x})=\mathcal{L}_{X}(\bar{x})$. Due to Remark 3.7, the validity of MPDC-LICQ also guarantees $\mathcal{T}_{X}(\bar{x})=\mathcal{L}_{X}(\bar{x})$, i.e. $\mathcal{T}_{X}^{b}(\bar{x})=\mathcal{T}_{X}(\bar{x})$ follows, and this yields the claim.

Lemma 3.11. Let $\bar{x} \in X$ be a feasible point of (MPDC) where MPDC-LICQ is valid. Then, for each $d \in \mathcal{T}_{X}(\bar{x})$, we have

$$
\mathcal{T}_{X}^{2}(\bar{x} ; d)=\left\{h \in \mathbb{R}^{n} \mid \nabla F(\bar{x}) h+\nabla^{2} F(\bar{x})[d, d] \in \mathcal{T}_{\mathcal{T}_{D}(F(\bar{x}))}(\nabla F(\bar{x}) d)\right\} .
$$

Furthermore, $\mathcal{T}_{X}^{2}(\bar{x} ; d)$ is nonempty and $X$ is parabolically derivable at $\bar{x}$ in direction $d$.

Proof. First, we note that the formula for the outer second-order tangent set follows from Lemma 2.5 noting that $\mathcal{T}_{D}^{2}(F(\bar{x}) ; \nabla F(\bar{x}) d)=\mathcal{T}_{\mathcal{T}_{D}(F(\bar{x}))}(\nabla F(\bar{x}) d)$ holds due to Lemma 2.4 while observing that the validity of MPDC-LICQ particularly yields that MSCQ is valid for (MPDC) at $\bar{x}$. For later use, we would like to mention that this implies $\mathcal{T}_{X}(\bar{x})=\mathcal{L}_{X}(\bar{x})$ as well, see Remark 3.7.

Next, let us show that $\mathcal{T}_{X}^{2}(\bar{x} ; d)$ is nonempty. Due to validity of $d \in \mathcal{L}_{X}(\bar{x})$, the set $\mathcal{T}_{\mathcal{T}_{D}(F(\bar{x}))}(\nabla F(\bar{x}) d)=\mathcal{T}_{D}^{2}(F(\bar{x}) ; \nabla F(\bar{x}) d)$ cannot be empty. We fix an arbitrary vector $r \in \mathcal{T}_{D}^{2}(F(\bar{x}) ; \nabla F(\bar{x}) d)$ and observe by means of Lemma 2.4 that

$$
\{r\}+\bigcap_{i \in I(\bar{x})} \mathcal{T}_{D_{i}}(F(\bar{x}))^{\circ \perp} \subset \mathcal{T}_{D}^{2}(F(\bar{x}) ; \nabla F(\bar{x}) d)=\mathcal{T}_{\mathcal{T}_{D}(F(\bar{x}))}(\nabla F(\bar{x}) d)
$$


holds true. By validity of MPDC-LICQ and Lemma 3.3, we now obtain

$$
\nabla F(\bar{x}) \mathbb{R}^{n}+\mathcal{T}_{\mathcal{T}_{D}(F(\bar{x}))}(\nabla F(\bar{x}) d)=\mathbb{R}^{m} .
$$

Particularly, we find some vector $h \in \mathbb{R}^{n}$ and some $w \in \mathcal{T}_{\mathcal{T}_{D}(F(\bar{x}))}(\nabla F(\bar{x}) d)$ such that we have $\nabla F(\bar{x}) h+w=\nabla^{2} F(\bar{x})[d, d]$, i.e. $-h \in \mathcal{T}_{X}^{2}(\bar{x} ; d)$ is valid.

Exploiting similar arguments as provided in the proof of Lemma 2.5 while observing that $D$ is parabolically derivable due to Lemma 2.4 , we can show

$$
\mathcal{T}_{X}^{b, 2}(\bar{x} ; d)=\left\{h \in \mathbb{R}^{n} \mid \nabla F(\bar{x}) h+\nabla^{2} F(\bar{x})[d, d] \in \mathcal{T}_{\mathcal{T}_{D}(F(\bar{x}))}(\nabla F(\bar{x}) d)\right\},
$$

i.e. $X$ is parabolically derivable at $\bar{x}$ in direction $d \in \mathcal{T}_{X}(\bar{x})$.

\section{Second-order optimality conditions and MPDC-LICQ}

Recall that for each feasible point $\bar{x} \in X$ of (MPDC), $\mathcal{L}_{X}(\bar{x})$ denotes the linearization cone to $X$ at $\bar{x}$ and has been defined in (2). For later use, we introduce the so-called critical cone to $X$ at $\bar{x}$ by means of

$$
\mathcal{C}_{X}(\bar{x}):=\left\{d \in \mathcal{L}_{X}(\bar{x}) \mid \nabla f(\bar{x}) \cdot d \leq 0\right\} .
$$

Furthermore, we will exploit the so-called Lagrangian function $L: \mathbb{R}^{n} \times \mathbb{R}^{m} \rightarrow \mathbb{R}$ of (MPDC) which is given as stated below:

$$
\forall(x, \lambda) \in \mathbb{R}^{n} \times \mathbb{R}^{m}: \quad L(x, \lambda):=f(x)+F(x) \cdot \lambda .
$$

Finally, let us introduce

$$
S(\bar{x}):=\left\{\lambda \in \bigcap_{i \in I(\bar{x})} \widehat{\mathcal{N}}_{D_{i}}(F(\bar{x})) \mid \nabla_{x} L(\bar{x}, \lambda)=0^{n}\right\}
$$

the set of all multipliers which solve the S-stationarity system associated with (MPDC) at $\bar{x}$. Clearly, $\bar{x}$ is an S-stationary point of (MPDC) if and only if $S(\bar{x})$ is nonempty.

Lemma 4.1. Let $\bar{x} \in X$ be an S-stationary point of (MPDC). Then, we have

$$
\forall \lambda \in S(\bar{x}): \quad \mathcal{C}_{X}(\bar{x})=\left\{d \in \mathbb{R}^{n} \mid \nabla F(\bar{x}) d \in \mathcal{T}_{D}(F(\bar{x})) \cap\{\lambda\}^{\perp}\right\} .
$$

Proof. For each $d \in \mathcal{C}_{X}(\bar{x})$ and $\lambda \in S(\bar{x})$, we obtain

$$
0 \geq \nabla f(\bar{x}) \cdot d=\left(-\nabla F(\bar{x})^{\top} \lambda\right) \cdot d=-(\underbrace{\nabla F(\bar{x}) d}_{\in \mathcal{T}_{D}(F(\bar{x}))}) \cdot \lambda \geq 0
$$

from $\lambda \in \bigcap_{i \in I(\bar{x})} \widehat{\mathcal{N}}_{D_{i}}(F(\bar{x}))=\widehat{\mathcal{N}}_{D}(F(\bar{x}))=\mathcal{T}_{D}(F(\bar{x}))^{\circ}$, see Lemma 2.2. This yields $\nabla F(\bar{x}) d \in\{\lambda\}^{\perp}$ and shows the inclusion $\subset$.

If, on the other hand, $d \in \mathbb{R}^{n}$ satisfies $\nabla F(\bar{x}) d \in \mathcal{T}_{D}(F(\bar{x})) \cap\{\lambda\}^{\perp}$ for some $\lambda \in S(\bar{x})$, then we have $d \in \mathcal{L}_{X}(\bar{x})$ by definition of the linearization cone and

$$
0=(\nabla F(\bar{x}) d) \cdot \lambda=\left(\nabla F(\bar{x})^{\top} \lambda\right) \cdot d=-\nabla f(\bar{x}) \cdot d
$$

which yields $d \in \mathcal{C}_{X}(\bar{x})$. 
Using the theory on second-order tangent sets provided earlier, we are now in position to state a second-order necessary optimality condition for (MPDC) under validity of MPDC-LICQ. Thus, our approach is closely related to the approaches used in Bonnans and Shapiro [2000], Christof and Wachsmuth [2018], Penot [1998], Rockafellar and Wets [1998] for the derivation of second-order necessary optimality conditions for different classes of mathematical programs in the finite- and infinite-dimensional setting. It seems to be worth mentioning that, in contrast to [Hoheisel and Kanzow, 2007, Theorem 4.3] where a second-order necessary optimality conditions for MPVCs is shown, we do not use an implicit function argument for our proof. Some parts of the upcoming theorem's proof are inspired by [Christof and Wachsmuth, 2018, Lemma 5.8].

Theorem 4.2. Let $\bar{x} \in X$ be a locally optimal solution of (MPDC) where $M P D C-L I C Q$ is valid. Then, we have

$$
\forall d \in \mathcal{C}_{X}(\bar{x}): \quad d^{\top} \nabla_{x x}^{2} L(\bar{x}, \bar{\lambda}) d \geq 0
$$

where $\bar{\lambda} \in S(\bar{x})$ is the uniquely determined multiplier which solves the S-stationarity system associated with $\bar{x}$, see Theorem 3.8.

Proof. First, we will prove the correctness of

$$
\forall d \in \mathcal{C}_{X}(\bar{x}) \forall h \in \mathcal{T}_{X}^{2}(\bar{x} ; d): \quad \nabla f(\bar{x}) \cdot h+d^{\top} \nabla^{2} f(\bar{x}) d \geq 0 .
$$

Therefore, fix $d \in \mathcal{C}_{X}(\bar{x})$ and $h \in \mathcal{T}_{X}^{2}(\bar{x} ; d)$. Then, we find sequences $\left\{t_{k}\right\}_{k \in \mathbb{N}} \subset \mathbb{R}_{+}$ and $\left\{h_{k}\right\}_{k \in \mathbb{N}} \subset \mathbb{R}^{n}$ such that $t_{k} \downarrow 0, h_{k} \rightarrow h$, and $\bar{x}+t_{k} d+\frac{1}{2} t_{k}^{2} h_{k} \in X$ for all $k \in \mathbb{N}$. Performing a second-order Taylor expansion of $f$ at $\bar{x}$ yields

$$
f\left(\bar{x}+t_{k} d+\frac{1}{2} t_{k}^{2} h_{k}\right)=f(\bar{x})+t_{k} \nabla f(\bar{x}) \cdot d+\frac{1}{2} t_{k}^{2}\left(\nabla f(\bar{x}) \cdot h_{k}+d^{\top} \nabla^{2} f(\bar{x}) d\right)+o\left(t_{k}^{2}\right)
$$

for all $k \in \mathbb{N}$. Noting that we have $f\left(\bar{x}+t_{k} d+\frac{1}{2} t_{k}^{2} h_{k}\right) \geq f(\bar{x})$ for sufficiently large $k \in \mathbb{N}$ from the local optimality of $\bar{x}$ for (MPDC) while $\nabla f(\bar{x}) \cdot d \leq 0$ holds by definition of the critical cone, we obtain

$$
0 \leq \frac{2}{t_{k}^{2}}\left(f\left(\bar{x}+t_{k} d+\frac{1}{2} t_{k}^{2} h_{k}\right)-f(\bar{x})-t_{k} \nabla f(\bar{x}) \cdot d\right)=\nabla f(\bar{x}) \cdot h_{k}+d^{\top} \nabla^{2} f(\bar{x}) d+2 \frac{o\left(t_{k}^{2}\right)}{t_{k}^{2}}
$$

for sufficiently large $k \in \mathbb{N}$. Thus, taking the limit $k \rightarrow \infty$ yields (8).

Due to validity of MPDC-LICQ, (8) implies that

$$
\inf \left\{\nabla f(\bar{x}) \cdot h \mid \nabla F(\bar{x}) h \in \mathcal{T}_{\mathcal{T}_{D}(F(\bar{x}))}(\nabla F(\bar{x}) d)-\left\{\nabla^{2} F(\bar{x})[d, d]\right\}\right\}+d^{\top} \nabla^{2} f(\bar{x}) d \geq 0
$$

holds true for all $d \in \mathcal{C}_{X}(\bar{x})$, see Lemma 3.11. By definition of S-stationarity, we have $\nabla f(\bar{x})=-\nabla F(\bar{x})^{\top} \bar{\lambda}$ which yields

$$
\inf \left\{\begin{array}{l|l}
-\bar{\lambda} \cdot w & \begin{array}{l}
w \in \mathcal{T}_{\mathcal{T}_{D}(F(\bar{x}))}(\nabla F(\bar{x}) d)-\left\{\nabla^{2} F(\bar{x})[d, d]\right\} \\
w \in \nabla F(\bar{x}) \mathbb{R}^{n}
\end{array}
\end{array}\right\}+d^{\top} \nabla^{2} f(\bar{x}) d \geq 0
$$


for each $d \in \mathcal{C}_{X}(\bar{x})$.

Due to validity of MPDC-LICQ, for each $w \in \mathcal{T}_{\mathcal{T}_{D}(F(\bar{x}))}(\nabla F(\bar{x}) d)-\left\{\nabla^{2} F(\bar{x})[d, d]\right\}$, we find $v \in \mathbb{R}^{n}$ and $\ell \in \bigcap_{i \in I(\bar{x})} \mathcal{T}_{D_{i}}(F(\bar{x}))^{\circ \perp}$ such that $w=\nabla F(\bar{x}) v-\ell$ holds true, see Lemma 3.3. Noting that we have

$$
\bigcap_{i \in I(\bar{x})} \mathcal{T}_{D_{i}}(F(\bar{x}))^{\circ \perp}=\bigcap_{i \in I(\bar{x})} \widehat{\mathcal{N}}_{D_{i}}(F(\bar{x}))^{\perp} \subset\left(\bigcap_{i \in I(\bar{x})} \widehat{\mathcal{N}}_{D_{i}}(F(\bar{x}))\right)^{\perp}=\widehat{\mathcal{N}}_{D}(F(\bar{x}))^{\perp},
$$

see Lemma 2.2, the relation $\bar{\lambda} \cdot \ell=0$ follows from $\bar{\lambda} \in \widehat{\mathcal{N}}_{D}(F(\bar{x}))$. Furthermore, we infer

$$
\begin{aligned}
\nabla F(\bar{x}) v=w+\ell & \in \mathcal{T}_{\mathcal{T}_{D}(F(\bar{x}))}(\nabla F(\bar{x}) d)-\left\{\nabla^{2} F(\bar{x})[d, d]\right\}+\bigcap_{i \in I(\bar{x})} \mathcal{T}_{D_{i}}(F(\bar{x}))^{\circ \perp} \\
& =\mathcal{T}_{D}^{2}(F(\bar{x}) ; \nabla F(\bar{x}) d)+\bigcap_{i \in I(\bar{x})} \mathcal{T}_{D_{i}}(F(\bar{x}))^{\circ \perp}-\left\{\nabla^{2} F(\bar{x})[d, d]\right\} \\
& \subset \mathcal{T}_{D}^{2}(F(\bar{x}) ; \nabla F(\bar{x}) d)-\left\{\nabla^{2} F(\bar{x})[d, d]\right\} \\
& =\mathcal{T}_{\mathcal{T}_{D}(F(\bar{x}))}(\nabla F(\bar{x}) d)-\left\{\nabla^{2} F(\bar{x})[d, d]\right\}
\end{aligned}
$$

from Lemma 2.4. Summarizing these considerations, we have shown $\nabla F(\bar{x}) v \in \nabla F(\bar{x}) \mathbb{R}^{n}$ and $\nabla F(\bar{x}) v \in \mathcal{T}_{\mathcal{T}_{D}(F(\bar{x}))}(\nabla F(\bar{x}) d)-\left\{\nabla^{2} F(\bar{x})[d, d]\right\}$. Furthermore, we obtain the relation $-\bar{\lambda} \cdot w=-\bar{\lambda}(\nabla F(\bar{x}) v-\ell)=-\bar{\lambda} \cdot(\nabla F(\bar{x}) v)$. This leads to

$$
\begin{aligned}
& \inf \left\{\begin{array}{l}
-\bar{\lambda} \cdot w \mid \begin{array}{l}
w \in \mathcal{T}_{\mathcal{T}_{D}(F(\bar{x}))}(\nabla F(\bar{x}) d)-\left\{\nabla^{2} F(\bar{x})[d, d]\right\} \\
w \in \nabla F(\bar{x}) \mathbb{R}^{n}
\end{array} \\
\leq \inf \left\{-\bar{\lambda} \cdot w \mid w \in \mathcal{T}_{\mathcal{T}_{D}(F(\bar{x}))}(\nabla F(\bar{x}) d)-\left\{\nabla^{2} F(\bar{x})[d, d]\right\}\right\} .
\end{array}\right.
\end{aligned}
$$

The converse inequality, however, is trivial. Thus, equality holds for the optimal values of the above programs and we obtain

$$
\inf \left\{-\bar{\lambda} \cdot w \mid w \in \mathcal{T}_{\mathcal{T}_{D}(F(\bar{x}))}(\nabla F(\bar{x}) d)-\left\{\nabla^{2} F(\bar{x})[d, d]\right\}\right\}+d^{\top} \nabla^{2} f(\bar{x}) d \geq 0
$$

for each $d \in \mathcal{C}_{X}(\bar{x})$ from (9). Clearly, we have

$$
\begin{aligned}
\inf \left\{-\bar{\lambda} \cdot w \mid w \in \mathcal{T}_{\mathcal{T}_{D}(F(\bar{x}))}(\nabla F(\bar{x}) d)-\left\{\nabla^{2} F(\bar{x})[d, d]\right\}\right\} \\
\quad=\inf \left\{-\bar{\lambda} \cdot w \mid w \in \mathcal{T}_{\mathcal{T}_{D}(F(\bar{x}))}(\nabla F(\bar{x}) d)\right\}+\bar{\lambda} \cdot \nabla^{2} F(\bar{x})[d, d] .
\end{aligned}
$$

Finally, we note that

$$
\begin{aligned}
& \mathcal{T}_{\mathcal{T}_{D}(F(\bar{x}))}(\nabla F(\bar{x}) d)=\bigcup_{i \in I(\bar{x})} \mathcal{T}_{\mathcal{T}_{D_{i}}(F(\bar{x}))}(\nabla F(\bar{x}) d) \\
& \quad=\bigcup_{i \in I(\bar{x})} \mathcal{T}_{D_{i}}(F(\bar{x}))-\operatorname{cone}\{\nabla F(\bar{x}) d\}=\mathcal{T}_{D}(F(\bar{x}))-\operatorname{cone}\{\nabla F(\bar{x}) d\}
\end{aligned}
$$

holds true invoking Lemma 2.2 while noticing that the sets $\mathcal{T}_{D_{i}}(F(\bar{x})), i \in I(\bar{x})$, are closed, convex, polyhedral cones. Thus, for each $w \in \mathcal{T}_{\mathcal{T}_{D}(F(\bar{x}))}(\nabla F(\bar{x}) d)$, we find a vector 
$r \in \mathcal{T}_{D}(F(\bar{x}))$ and $\alpha \geq 0$ such that $w=r-\alpha \nabla F(\bar{x}) d$ holds. Recalling $\bar{\lambda} \in \widehat{\mathcal{N}}_{D}(F(\bar{x}))$ and $d \in \mathcal{C}_{X}(\bar{x})$, we have

$$
-\bar{\lambda} \cdot w=-\bar{\lambda} \cdot(r-\alpha \nabla F(\bar{x}) d) \geq \alpha\left(\nabla F(\bar{x})^{\top} \bar{\lambda}\right) \cdot d=\alpha(-\nabla f(\bar{x})) \cdot d \geq 0
$$

by definition of S-stationarity, i.e.

$$
\inf \left\{-\bar{\lambda} \cdot w \mid w \in \mathcal{T}_{\mathcal{T}_{D}(F(\bar{x}))}(\nabla F(\bar{x}) d)\right\}+\bar{\lambda} \cdot \nabla^{2} F(\bar{x})[d, d]=\bar{\lambda} \cdot \nabla^{2} F(\bar{x})[d, d]
$$

follows for each $d \in \mathcal{C}_{X}(\bar{x})$. Combining this with the above arguments, the desired result follows from (10) by definition of the Lagrangian function. This completes the proof.

The above result can be seen as a particular instance of [Gfrerer, 2014, Theorem 3.3] where a second-order necessary optimality condition for (MPDC) has been derived using a completely different approach via the variational concepts of the directional limiting normal cone and directional metric subregularity. One can easily check that by demanding validity of MPDC-LICQ at a given local minimizer of (MPDC), the assumptions of [Gfrerer, 2014, Theorem 3.3] hold as well, i.e. the assumptions of Theorem 4.2 are more restrictive. On the other hand, one has to mention that checking validity of MPDC-LICQ and noting that this implies that there is only one S-stationary multiplier, the secondorder necessary optimality condition from Theorem 4.2 seems to be much easier to verify than the one from Gfrerer [2014].

Next, we state a second-order sufficient optimality condition for (MPDC). Although this result follows from [Gfrerer, 2014, Theorem 3.21], we provide a completely elementary and simple proof here which generalizes a well-known strategy which has been used to verify second-order sufficient optimality conditions for NLPs, MPCCs, MPVCs, and CCMPs in the past.

Theorem 4.3. Let $\bar{x} \in X$ be an S-stationary point of (MPDC) where the condition

$$
\forall d \in \mathcal{C}_{X}(\bar{x}) \backslash\left\{0^{n}\right\} \exists \lambda \in S(\bar{x}): \quad d^{\top} \nabla_{x x}^{2} L(\bar{x}, \lambda) d>0
$$

holds. Then, there are constants $\varepsilon>0$ and $C>0$ such that the following quadraticgrowth-condition is valid:

$$
\forall x \in X \cap \mathbb{B}^{\varepsilon}(\bar{x}): \quad f(x) \geq f(\bar{x})+C\|x-\bar{x}\|_{2}^{2} .
$$

Particularly, $\bar{x}$ is a strict local minimizer of (MPDC).

Proof. Assume on the contrary that there is a sequence $\left\{x_{k}\right\}_{k \in \mathbb{N}} \subset X$ converging to $\bar{x}$ such that

$$
\forall k \in \mathbb{N}: \quad f\left(x_{k}\right)<f(\bar{x})+\frac{1}{k}\left\|x_{k}-\bar{x}\right\|_{2}^{2}
$$

holds true. Set $t_{k}:=\left\|x_{k}-\bar{x}\right\|_{2}>0$ and observe that $\left\{\left(x_{k}-\bar{x}\right) / t_{k}\right\}_{k \in \mathbb{N}}$ is bounded. We assume w.l.o.g. that $\left(x_{k}-\bar{x}\right) / t_{k} \rightarrow d$ holds for some $d \in \mathbb{R}^{n} \backslash\left\{0^{n}\right\}$. By construction, $d \in \mathcal{T}_{X}(\bar{x}) \subset \mathcal{L}_{X}(\bar{x})$ is guaranteed. For each $k \in \mathbb{N}$, we find $\xi_{k} \in \operatorname{conv}\left\{\bar{x} ; x_{k}\right\}$ which satisfies $f\left(x_{k}\right)-f(\bar{x})=\nabla f\left(\xi_{k}\right) \cdot\left(x_{k}-\bar{x}\right)$ by means of the mean value theorem. Dividing 
by $t_{k}$ and taking the limit $k \rightarrow \infty$ while observing that $\nabla f: \mathbb{R}^{n} \rightarrow \mathbb{R}^{n}$ is continuous, we have

$$
\nabla f(\bar{x}) \cdot d=\lim _{k \rightarrow \infty} \nabla f\left(\xi_{k}\right) \cdot \frac{x_{k}-\bar{x}}{t_{k}}=\lim _{k \rightarrow \infty} \frac{f\left(x_{k}\right)-f(\bar{x})}{t_{k}} \leq \lim _{k \rightarrow \infty} \frac{1}{k}\left\|x_{k}-\bar{x}\right\|_{2}=0 .
$$

This yields $d \in \mathcal{C}_{X}(\bar{x}) \backslash\left\{0^{n}\right\}$.

Choose $\lambda \in S(\bar{x})$ arbitrarily. Then, we have $\lambda \in \bigcap_{i \in I(\bar{x})} \widehat{\mathcal{N}}_{D_{i}}(F(\bar{x}))$. For sufficiently large $k \in \mathbb{N}, I\left(x_{k}\right) \subset I(\bar{x})$ holds true. Thus, for sufficiently large $k \in \mathbb{N}$ and $i \in I\left(x_{k}\right)$, we have $\lambda \in \widehat{\mathcal{N}}_{D_{i}}(F(\bar{x}))=\left(D_{i}-\{F(\bar{x})\}\right)^{\circ}$ which shows $\left(F\left(x_{k}\right)-F(\bar{x})\right) \cdot \lambda \leq 0$. This yields

$$
f(\bar{x})>f\left(x_{k}\right)-\frac{1}{k}\left\|x_{k}-\bar{x}\right\|_{2}^{2} \geq f\left(x_{k}\right)+\left(F\left(x_{k}\right)-F(\bar{x})\right) \cdot \lambda-\frac{1}{k}\left\|x_{k}-\bar{x}\right\|_{2}^{2}
$$

for sufficiently large $k \in \mathbb{N}$. Rearranging some terms and applying Taylor's theorem, we derive

$$
\begin{aligned}
L(\bar{x}, \lambda) & >L\left(x_{k}, \lambda\right)-\frac{1}{k}\left\|x_{k}-\bar{x}\right\|_{2}^{2} \\
& =L(\bar{x}, \lambda)+\nabla_{x} L(\bar{x}, \lambda)\left(x_{k}-\bar{x}\right)+\frac{1}{2}\left(x_{k}-\bar{x}\right)^{\top} \nabla_{x x}^{2} L(\bar{x}, \lambda)\left(x_{k}-\bar{x}\right)+o\left(\left\|x_{k}-\bar{x}\right\|_{2}^{2}\right) .
\end{aligned}
$$

Now, we exploit the choice $\lambda \in S(\bar{x})$ in order to infer

$$
0>\frac{1}{2}\left(x_{k}-\bar{x}\right)^{\top} \nabla_{x x}^{2} L(\bar{x}, \lambda)\left(x_{k}-\bar{x}\right)+o\left(\left\|x_{k}-\bar{x}\right\|_{2}^{2}\right)
$$

for sufficiently large $k \in \mathbb{N}$. Division by $t_{k}^{2}$ and taking the limit $k \rightarrow \infty$ yield

$$
0 \geq \frac{1}{2} d^{\top} \nabla_{x x}^{2} L(\bar{x}, \lambda) d
$$

which contradicts the theorem's assumptions since we have shown $d \in \mathcal{C}_{X}(\bar{x}) \backslash\left\{0^{n}\right\}$ while $\lambda \in S(\bar{x})$ was arbitrarily chosen. This completes the proof.

The above result justifies the following definition.

Definition 4.4. Let $\bar{x} \in X$ be an S-stationary point of (MPDC). Then, the MPDCtailored second-order sufficient condition (MPDC-SOSC for short) holds at $\bar{x}$ if and only if (11) is valid.

The upcoming considerations will show that S-stationary points of (MPDC), where both MPDC-LICQ and MPDC-SOSC are valid, are locally isolated w.r.t. primal and dual variables. This property does not generally follow from the second-order growth condition as [Guo et al., 2013, Example 4.1], which has been stated in the context of MPCCs, indicates. For the validation of the upcoming result, we generalize the proof of [Guo et al., 2013, Theorem 4.1].

Theorem 4.5. Let $\bar{x} \in X$ be an S-stationary point of (MPDC) where MPDC-LICQ and $M P D C-S O S C$ are valid. Then, there is some $\varepsilon>0$ such that we have

$$
\forall x \in X \cap \mathbb{B}^{\varepsilon}(\bar{x}): \quad \lambda \in S(x) \Longrightarrow x=\bar{x}, \lambda=\bar{\lambda}
$$

where $\bar{\lambda}$ is the uniquely determined vector from $S(\bar{x})$. 
Proof. Due to validity of MPDC-LICQ, the S-stationarity multiplier $\bar{\lambda}$ associated with $\bar{x}$ is indeed uniquely determined, see Theorem 3.8. Assume on the contrary, that we can find a sequence $\left\{x_{k}\right\}_{k \in \mathbb{N}} \subset X \backslash\{\bar{x}\}$ of feasible and S-stationary points of (MPDC) converging to $\bar{x}$. Then, we find $\lambda_{k} \in S\left(x_{k}\right)$ for each $k \in \mathbb{N}$.

Suppose that $\left\{\lambda_{k}\right\}_{k \in \mathbb{N}}$ is not bounded, i.e. we can assume w.l.o.g. that $\left\|\lambda_{k}\right\|_{2} \rightarrow \infty$ holds as $k \rightarrow \infty$. Thus, we can define $\tilde{\lambda}_{k}:=\lambda_{k} /\left\|\lambda_{k}\right\|_{2}$ for sufficiently large $k \in \mathbb{N}$ and due to the boundedness of $\left\{\tilde{\lambda}_{k}\right\}_{k \in \mathbb{N}}$, we may assume w.l.o.g. that this sequence converges to some nonvanishing vector $\tilde{\lambda} \in \mathbb{R}^{m}$. Furthermore, we have

$$
\nabla F(\bar{x})^{\top} \tilde{\lambda}=\lim _{k \rightarrow \infty} \nabla F\left(x_{k}\right)^{\top} \tilde{\lambda}_{k}=\lim _{k \rightarrow \infty} \frac{1}{\left\|\lambda_{k}\right\|_{2}} \underbrace{\left(\nabla f\left(x_{k}\right)+\nabla F\left(x_{k}\right)^{\top} \lambda_{k}\right)}_{=0^{n}}=0^{n}
$$

by continuity of $\nabla f: \mathbb{R}^{n} \rightarrow \mathbb{R}^{n}$ and $\nabla F: \mathbb{R}^{n} \rightarrow \mathbb{R}^{m \times n}$ as well as $\lambda_{k} \in S\left(x_{k}\right)$. On the other hand, the inclusion $I\left(x_{k}\right) \subset I(\bar{x})$ is valid for all sufficiently large $k \in \mathbb{N}$ and, clearly, $I\left(x_{k}\right) \neq \varnothing$ is true as well since $x_{k}$ is feasible to (MPDC) for each $k \in \mathbb{N}$. Noting that there are only finitely many indices in $I(\bar{x})$, there must exist some $i_{0} \in I(\bar{x})$ such that $i_{0} \in I\left(x_{k_{l}}\right)$ for all $l \in \mathbb{N}$ holds along a subsequence $\left\{x_{k_{l}}\right\}_{l \in \mathbb{N}}$ of $\left\{x_{k}\right\}_{k \in \mathbb{N}}$. The definition of S-stationarity and the fact that the Fréchet normal cone is a cone yield $\tilde{\lambda}_{k_{l}} \in \widehat{\mathcal{N}}_{D_{i_{0}}}\left(F\left(x_{k_{l}}\right)\right)$. Now, the continuity of $F$ can be used to infer

$$
\tilde{\lambda} \in \mathcal{N}_{D_{i_{0}}}(F(\bar{x}))=\widehat{\mathcal{N}}_{D_{i_{0}}}(F(\bar{x})) \subset \sum_{i \in I(\bar{x})} \operatorname{span} \widehat{\mathcal{N}}_{D_{i}}(F(\bar{x})) .
$$

Keeping $\nabla F(\bar{x})^{\top} \tilde{\lambda}=0^{n}$ and $\tilde{\lambda} \neq 0^{m}$ in mind, this contradicts MPDC-LICQ.

Due to the above arguments, we may assume w.l.o.g. that $\left\{\lambda_{k}\right\}_{k \in \mathbb{N}}$ converges to some $\lambda \in \mathbb{R}^{m}$. Similar arguments as above show the existence of $i_{0} \in I(\bar{x})$ such that $\lambda \in \widehat{\mathcal{N}}_{D_{i_{0}}}(F(\bar{x}))$ holds true. Moreover, from $\nabla f\left(x_{k}\right)+\nabla F\left(x_{k}\right)^{\top} \lambda_{k}=0^{n}$ we obtain $\nabla f(\bar{x})+\nabla F(\bar{x})^{\top} \lambda=0^{n}$ since $f$ and $F$ possess continuous derivatives. Keeping $\nabla f(\bar{x})+\nabla F(\bar{x})^{\top} \bar{\lambda}=0^{n}$ in mind, we derive $\nabla F(\bar{x})^{\top}(\bar{\lambda}-\lambda)=0^{n}$. Moreover,

$$
\begin{aligned}
\bar{\lambda}-\lambda & \in\left(\bigcap_{i \in I(\bar{x})} \widehat{\mathcal{N}}_{D_{i}}(F(\bar{x}))\right)-\widehat{\mathcal{N}}_{D_{i_{0}}}(F(\bar{x})) \\
& \subset \widehat{\mathcal{N}}_{D_{i_{0}}}(F(\bar{x}))-\widehat{\mathcal{N}}_{D_{i_{0}}}(F(\bar{x}))=\operatorname{span} \widehat{\mathcal{N}}_{D_{i_{0}}}(F(\bar{x})) \subset \sum_{i \in I(\bar{x})} \operatorname{span} \widehat{\mathcal{N}}_{D_{i}}(F(\bar{x}))
\end{aligned}
$$

follows, and by validity of MPDC-LICQ, $\lambda=\bar{\lambda}$ is obtained.

We set $t_{k}:=\left\|x_{k}-\bar{x}\right\|_{2}>0$ and observe that $\left\{\left(x_{k}-\bar{x}\right) / t_{k}\right\}_{k \in \mathbb{N}}$ is a bounded sequence that converges w.l.o.g. to some nonvanishing direction $d \in \mathbb{R}^{n}$. Since $\left\{x_{k}\right\}_{k \in \mathbb{N}} \subset X$ holds, we infer $d \in \mathcal{T}_{X}(\bar{x}) \backslash\left\{0^{n}\right\} \subset \mathcal{L}_{X}(\bar{x}) \backslash\left\{0^{n}\right\}$. From $\lambda_{k} \in \widehat{\mathcal{N}}_{D}\left(F\left(x_{k}\right)\right)$ for all $k \in \mathbb{N}, x_{k} \rightarrow \bar{x}$, and $\lambda_{k} \rightarrow \bar{\lambda}$, we obtain $\lambda_{k} \cdot\left(F\left(x_{k}\right)-F(\bar{x})\right)=\bar{\lambda} \cdot\left(F\left(x_{k}\right)-F(\bar{x})\right)=0$ for all sufficiently large $k \in \mathbb{N}$, see Lemma 2.3. This yields

$$
(\nabla F(\bar{x}) d) \cdot \bar{\lambda}=\left(\lim _{k \rightarrow \infty} \frac{\nabla F(\bar{x})\left(x_{k}-\bar{x}\right)}{t_{k}}\right) \cdot \bar{\lambda}=\lim _{k \rightarrow \infty} \frac{\left(F\left(x_{k}\right)-F(\bar{x})\right) \cdot \bar{\lambda}}{t_{k}}=0,
$$


i.e. $F(\bar{x}) d \in\{\bar{\lambda}\}^{\perp}$ holds true. By means of Lemma 4.1, we deduce $d \in \mathcal{C}_{X}(\bar{x}) \backslash\left\{0^{n}\right\}$.

For each $k \in \mathbb{N}$, let us define a continuously differentiable function $\varphi_{k}:[0,1] \rightarrow \mathbb{R}$ by means of

$$
\begin{aligned}
\forall s \in[0,1]: \quad \varphi_{k}(s):= & \nabla_{x} L\left((1-s)(\bar{x}, \bar{\lambda})+s\left(x_{k}, \lambda_{k}\right)\right) \cdot\left(x_{k}-\bar{x}\right) \\
& -L\left((1-s) \bar{x}+s x_{k}, \lambda_{k}\right)+L\left((1-s) \bar{x}+s x_{k}, \bar{\lambda}\right) .
\end{aligned}
$$

Due to the above remarks, we have

$$
\begin{aligned}
\varphi_{k}(0) & =\nabla_{x} L(\bar{x}, \bar{\lambda}) \cdot\left(x_{k}-\bar{x}\right)-L\left(\bar{x}, \lambda_{k}\right)+L(\bar{x}, \bar{\lambda}) \\
& =\left(\bar{\lambda}-\lambda_{k}\right) \cdot F(\bar{x})=\left(\bar{\lambda}-\lambda_{k}\right) \cdot F\left(x_{k}\right) \\
& =\nabla_{x} L\left(x_{k}, \lambda_{k}\right) \cdot\left(x_{k}-\bar{x}\right)-L\left(x_{k}, \lambda_{k}\right)+L\left(x_{k}, \bar{\lambda}\right)=\varphi_{k}(1)
\end{aligned}
$$

for sufficiently large $k \in \mathbb{N}$. Due to $\varphi_{k}(0)=\varphi_{k}(1)$, we can apply Rolle's theorem in order to obtain the existence of $s_{k} \in(0,1)$ such that

$$
\begin{aligned}
0= & \varphi_{k}^{\prime}\left(s_{k}\right) \\
= & \left(x_{k}-\bar{x}\right)^{\top} \nabla_{x x}^{2} L\left(\left(1-s_{k}\right)(\bar{x}, \bar{\lambda})+s_{k}\left(x_{k}, \lambda_{k}\right)\right)\left(x_{k}-\bar{x}\right) \\
& \quad+\left(x_{k}-\bar{x}\right)^{\top} \nabla_{x, \lambda}^{2} L\left(\left(1-s_{k}\right)(\bar{x}, \bar{\lambda})+s_{k}\left(x_{k}, \lambda_{k}\right)\right)\left(\lambda_{k}-\bar{\lambda}\right) \\
& \quad-\nabla_{x} L\left(\left(1-s_{k}\right) \bar{x}+s_{k} x_{k}, \lambda_{k}\right) \cdot\left(x_{k}-\bar{x}\right)+\nabla_{x} L\left(\left(1-s_{k}\right) \bar{x}+s_{k} x_{k}, \bar{\lambda}\right) \cdot\left(x_{k}-\bar{x}\right) \\
= & \left(x_{k}-\bar{x}\right)^{\top} \nabla_{x x}^{2} L\left(\left(1-s_{k}\right)(\bar{x}, \bar{\lambda})+s_{k}\left(x_{k}, \lambda_{k}\right)\right)\left(x_{k}-\bar{x}\right) \\
& \quad+\left(x_{k}-\bar{x}\right) \cdot\left[\nabla F\left(\left(1-s_{k}\right) \bar{x}+s_{k} x_{k}\right)^{\top}\left(\lambda_{k}-\bar{\lambda}\right)\right] \\
& \quad-\left[\nabla F\left(\left(1-s_{k}\right) \bar{x}+s_{k} x_{k}\right)^{\top}\left(\lambda_{k}-\bar{\lambda}\right)\right] \cdot\left(x_{k}-\bar{x}\right) \\
= & \left(x_{k}-\bar{x}\right)^{\top} \nabla_{x x}^{2} L\left(\left(1-s_{k}\right)(\bar{x}, \bar{\lambda})+s_{k}\left(x_{k}, \lambda_{k}\right)\right)\left(x_{k}-\bar{x}\right)
\end{aligned}
$$

holds for all $k \in \mathbb{N}$ which are sufficiently large. Next, we observe that the relation $\left(1-s_{k}\right)(\bar{x}, \bar{\lambda})+s_{k}\left(x_{k}, \lambda_{k}\right) \rightarrow(\bar{x}, \bar{\lambda})$ holds true as $k \rightarrow \infty$. From above, it follows

$$
0=\left(\frac{x_{k}-\bar{x}}{t_{k}}\right)^{\top} \nabla_{x x}^{2} L\left(\left(1-s_{k}\right)(\bar{x}, \bar{\lambda})+s_{k}\left(x_{k}, \lambda_{k}\right)\right)\left(\frac{x_{k}-\bar{x}}{t_{k}}\right)
$$

for sufficiently large $k \in \mathbb{N}$, i.e. taking the limit $k \rightarrow \infty$ yields $0=d^{\top} \nabla_{x x}^{2} L(\bar{x}, \bar{\lambda}) d$. This, however, contradicts the validity of MPDC-SOSC since we already verified that $d \in \mathcal{C}_{X}(\bar{x}) \backslash\left\{0^{n}\right\}$ holds true. Thus, the proof is completed.

\section{Consequences for certain classes of disjunctive programs}

In this section, we are going to apply the obtained results to some prominent classes of disjunctive programs, namely MPCCs, MPVCs, CCMPs, and MPSCs in order to check how the above theory relates to existing results in the available literature on these problem classes. Throughout the section, we consider twice continuously differentiable functions $f: \mathbb{R}^{n} \rightarrow \mathbb{R}, g: \mathbb{R}^{n} \rightarrow \mathbb{R}^{p}, h: \mathbb{R}^{n} \rightarrow \mathbb{R}^{q}$, and $G, H: \mathbb{R}^{n} \rightarrow \mathbb{R}^{l}$. The component mappings of $g, h, G$, and $H$ will be denoted by $g_{j}: \mathbb{R}^{n} \rightarrow \mathbb{R}, j=1, \ldots, p, h_{j}: \mathbb{R}^{n} \rightarrow \mathbb{R}$, $j=1, \ldots, q$, and $G_{j}, H_{j}: \mathbb{R}^{n} \rightarrow \mathbb{R}, j=1, \ldots, l$, respectively. 


\subsection{Application to MPCCs}

A mathematical program with complementarity constraints is an optimization problem of the form

$$
\begin{aligned}
f(x) & \rightarrow \min & & \\
g_{j}(x) & \leq 0 & & j=1, \ldots, p \\
h_{j}(x) & =0 & & j=1, \ldots, q \\
0 \leq G_{j}(x) \perp H_{j}(x) & \geq 0 & & j=1, \ldots, l .
\end{aligned}
$$

Due to the frequent appearance of (MPCC) as an abstract model of real-world applications, this problem class has been studied intensively from the theoretical and numerical point of view during the last two decades, see e.g. Gfrerer [2014], Hoheisel et al. [2013], Luo et al. [1996], Outrata et al. [1998], Scheel and Scholtes [2000], Ye [2005] and the references therein.

In order to transfer (MPCC) into a program of type (MPDC), we introduce the sets $S_{1}^{\mathrm{CC}}:=\mathbb{R}_{+} \times\{0\}$ and $S_{2}^{\mathrm{CC}}:=\{0\} \times \mathbb{R}_{+}$as well as $\mathcal{J}:=\{1,2\}^{l}$. Next, we set

$$
\forall \alpha \in \mathcal{J}: \quad D_{\alpha}^{\mathrm{CC}}:=\mathbb{R}_{-}^{p} \times\left\{0^{q}\right\} \times \prod_{j=1}^{l} S_{\alpha_{j}}^{\mathrm{CC}}
$$

and $D^{\mathrm{CC}}:=\bigcup_{\alpha \in \mathcal{J}} D_{\alpha}^{\mathrm{CC}}$. Furthermore, we introduce $F: \mathbb{R}^{n} \rightarrow \mathbb{R}^{p+q+2 l}$ by means of

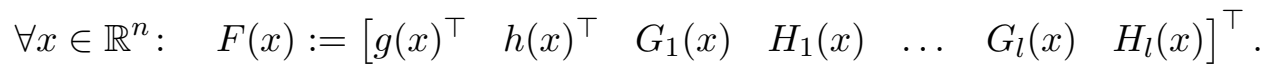

Then, the feasible set of (MPCC) is given by $X^{\mathrm{CC}}:=\left\{x \in \mathbb{R}^{n} \mid F(x) \in D^{\mathrm{CC}}\right\}$, see Figure 1 for an illustration. For a feasible point $\bar{x} \in X^{\mathrm{CC}}$ of (MPCC), let us introduce
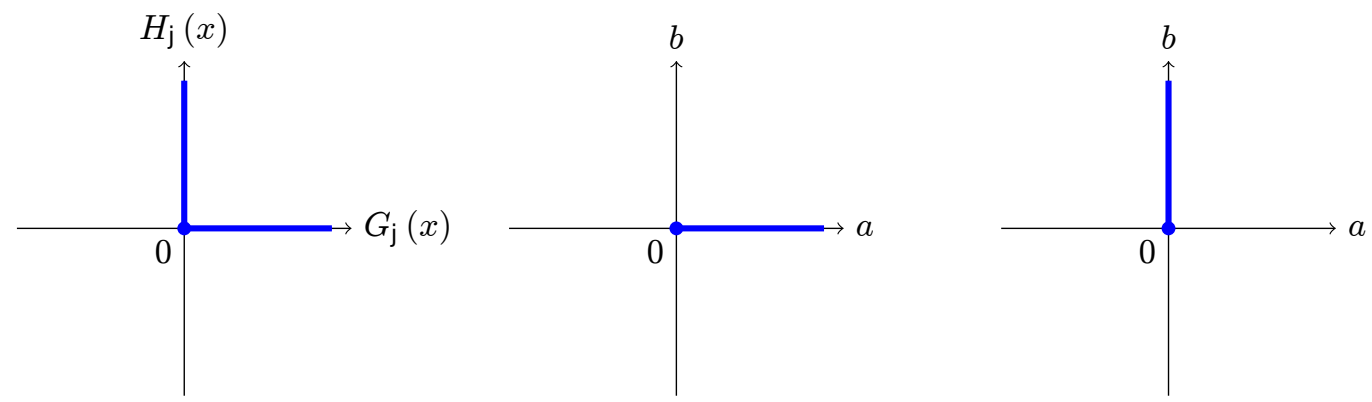

Figure 1: Geometric illustrations of $X^{\mathrm{CC}}$ (left), $S_{1}^{\mathrm{CC}}$ (middle), and $S_{2}^{\mathrm{CC}}$ (right), respectively.

the following well-known index sets:

$$
\begin{aligned}
I^{+0}(\bar{x}) & :=\left\{j \in\{1, \ldots, l\} \mid G_{j}(\bar{x})>0, H_{j}(\bar{x})=0\right\}, \\
I^{0+}(\bar{x}) & :=\left\{j \in\{1, \ldots, l\} \mid G_{j}(\bar{x})=0, H_{j}(\bar{x})>0\right\}, \\
I^{00}(\bar{x}) & :=\left\{j \in\{1, \ldots, l\} \mid G_{j}(\bar{x})=0, H_{j}(\bar{x})=0\right\} .
\end{aligned}
$$


We exploit the calculus rules for the tangent and Fréchet normal cone to Cartesian products of (convex) sets, see [Rockafellar and Wets, 1998, Proposition 6.41], in order to obtain

$$
\begin{aligned}
& \mathcal{T}_{D_{\alpha}^{\mathrm{CC}}}(F(\bar{x}))=\mathcal{T}_{\mathbb{R}_{-}^{p}}(g(\bar{x})) \times \mathcal{T}_{\left\{0^{q}\right\}}(h(\bar{x})) \times \prod_{j=1}^{l} \mathcal{T}_{S_{\alpha_{j}}^{\mathrm{CC}}}\left(\left(G_{j}(\bar{x}), H_{j}(\bar{x})\right)^{\top}\right) \\
& \widehat{\mathcal{N}}_{D_{\alpha}^{\mathrm{CC}}}(F(\bar{x}))=\widehat{\mathcal{N}}_{\mathbb{R}_{-}^{p}}(g(\bar{x})) \times \widehat{\mathcal{N}}_{\left\{0^{q}\right\}}(h(\bar{x})) \times \prod_{j=1}^{l} \widehat{\mathcal{N}}_{S_{\alpha_{j}}^{\mathrm{CC}}}\left(\left(G_{j}(\bar{x}), H_{j}(\bar{x})\right)^{\top}\right)
\end{aligned}
$$

for each $\alpha \in \mathcal{J}$. The tangent and Fréchet normal cones to the sets $\mathbb{R}_{-}^{p}$ and $\left\{0^{q}\right\}$ have been characterized in Example 3.2 already. A straightforward calculation shows

$$
\begin{aligned}
& \mathcal{T}_{S_{1}^{\mathrm{CC}}}\left(\left(G_{j}(\bar{x}), H_{j}(\bar{x})\right)^{\top}\right)= \begin{cases}\mathbb{R} \times\{0\} & j \in I^{+0}(\bar{x}), \\
\varnothing & j \in I^{0+}(\bar{x}), \\
\mathbb{R}_{+} \times\{0\} & j \in I^{00}(\bar{x}),\end{cases} \\
& \mathcal{T}_{S_{2}^{\mathrm{CC}}}\left(\left(G_{j}(\bar{x}), H_{j}(\bar{x})\right)^{\top}\right)= \begin{cases}\varnothing & j \in I^{+0}(\bar{x}), \\
\{0\} \times \mathbb{R} & j \in I^{0+}(\bar{x}), \\
\{0\} \times \mathbb{R}_{+} & j \in I^{00}(\bar{x}),\end{cases} \\
& \widehat{\mathcal{N}}_{S_{1}^{\mathrm{CC}}}\left(\left(G_{j}(\bar{x}), H_{j}(\bar{x})\right)^{\top}\right)= \begin{cases}\{0\} \times \mathbb{R} & j \in I^{+0}(\bar{x}), \\
\varnothing & j \in I^{0+}(\bar{x}), \\
\mathbb{R}_{-} \times \mathbb{R} & j \in I^{00}(\bar{x}),\end{cases} \\
& \widehat{\mathcal{N}}_{S_{2}^{\mathrm{CC}}}\left(\left(G_{j}(\bar{x}), H_{j}(\bar{x})\right)^{\top}\right)= \begin{cases}\varnothing & j \in I^{+0}(\bar{x}), \\
\mathbb{R} \times\{0\} & j \in I^{0+}(\bar{x}), \\
\mathbb{R} \times \mathbb{R}_{-} & j \in I^{00}(\bar{x}) .\end{cases}
\end{aligned}
$$

Using $I(\bar{x}):=\left\{\alpha \in \mathcal{J} \mid F(\bar{x}) \in D_{\alpha}^{\mathrm{CC}}\right\}$, we obtain the characterization

$$
\alpha \in I(\bar{x}) \Longleftrightarrow \forall j \in I^{+0}(\bar{x}): \alpha_{j}=1 \wedge \forall j \in I^{0+}(\bar{x}): \alpha_{j}=2
$$

for arbitrary $\alpha \in \mathcal{J}$. Thus, MPDC-LICQ from Definition 3.1 takes the following form for problem (MPCC) at the reference point $\bar{x}$ :

$$
\left.\begin{array}{l}
0^{n}=\nabla g(\bar{x})^{\top} \lambda+\nabla h(\bar{x})^{\top} \rho+\nabla G(\bar{x})^{\top} \mu+\nabla H(\bar{x})^{\top} \nu, \\
\forall j \notin I^{g}(\bar{x}): \lambda_{j}=0, \\
\forall j \in I^{+0}(\bar{x}): \mu_{j}=0, \\
\forall j \in I^{0+}(\bar{x}): \nu_{j}=0
\end{array}\right\} \Longrightarrow\left\{\begin{array}{l}
\lambda=0^{p}, \rho=0^{q}, \\
\mu=\nu=0^{l} .
\end{array}\right.
$$

Here, the appearing index set $I^{g}(\bar{x})$ has been defined in Example 3.2. The above condition is equivalent to the linear independence of the vectors from

$$
\left\{\nabla g_{j}(\bar{x}) \mid j \in I^{g}(\bar{x})\right\} \cup\left\{\nabla h_{j}(\bar{x}) \mid j \in\{1, \ldots, q\}\right\}
$$




$$
\cup\left\{\nabla G_{j}(\bar{x}) \mid j \in I^{0+}(\bar{x}) \cup I^{00}(\bar{x})\right\} \cup\left\{\nabla H_{j}(\bar{x}) \mid j \in I^{+0}(\bar{x}) \cup I^{00}(\bar{x})\right\} .
$$

This, however, is precisely the definition of the prominent constraint qualification MPCCLICQ, see e.g. [Ye, 2005, Definition 2.8]. A similar observation has been made in [Gfrerer, 2014, Section 4] using different arguments. The associated S-stationarity system from Definition 3.9 reads as

$$
\begin{aligned}
& 0^{n}=\nabla f(\bar{x})+\nabla g(\bar{x})^{\top} \lambda+\nabla h(\bar{x})^{\top} \rho+\nabla G(\bar{x})^{\top} \mu+\nabla H(\bar{x})^{\top} \nu, \\
& \lambda \geq 0^{p}, \forall j \notin I^{g}(\bar{x}): \lambda_{j}=0, \\
& \forall j \in I^{+0}(\bar{x}): \mu_{j}=0, \\
& \forall j \in I^{0+}(\bar{x}): \nu_{j}=0, \\
& \forall j \in I^{00}(\bar{x}): \mu_{j}, \nu_{j} \leq 0
\end{aligned}
$$

and equals the MPCC-tailored system of strong stationarity, see [Ye, 2005, Definition 2.7]. Using the above formulas for the appearing tangent cones, the linearization cone from (2) is given by

$$
\mathcal{L}_{X^{\mathrm{CC}}}(\bar{x})=\left\{d \in \mathbb{R}^{n} \mid \begin{array}{rl}
\nabla g_{j}(\bar{x}) \cdot d \leq 0 & j \in I^{g}(\bar{x}) \\
\nabla h_{j}(\bar{x}) \cdot d=0 & j \in\{1, \ldots, q\} \\
\nabla G_{j}(\bar{x}) \cdot d=0 \quad j \in I^{0+}(\bar{x}) \\
\nabla H_{j}(\bar{x}) \cdot d=0 \quad j \in I^{+0}(\bar{x}) \\
0 \leq \nabla G_{j}(\bar{x}) \cdot d \perp \nabla H(\bar{x}) \cdot d \geq 0 \quad j \in I^{00}(\bar{x})
\end{array}\right\}
$$

while the critical cone from (7) can be easily represented by means of Lemma 4.1 whenever the reference point $\bar{x}$ is S-stationary for (MPCC). As a consequence, Theorems 3.8, 4.2 and 4.3 recover results from the classical paper Scheel and Scholtes [2000] while the stability result from Theorem 4.5 can be found in slightly stronger form in [Guo et al., 2013, Theorem 4.1].

\subsection{Application to MPVCs}

An optimization problem of type

$$
\begin{aligned}
f(x) & \rightarrow \min & & \\
g_{j}(x) & \leq 0 & & j=1, \ldots, p \\
h_{j}(x) & =0 & & j=1, \ldots, q \\
H_{j}(x) & \geq 0 & & j=1, \ldots, l \\
G_{j}(x) H_{j}(x) & \leq 0 & & j=1, \ldots, l
\end{aligned}
$$

is called a mathematical program with vanishing constraints. The term vanishing reflects the observation that whenever a point $x \in \mathbb{R}^{n}$ satisfies $H_{j}(x)=0$ for some $j \in\{1, \ldots, l\}$, then the constraint $G_{j}(x) H_{j}(x) \leq 0$ is trivially satisfied. Problems of type (MPVC) arise when searching for the optimal design of a truss structure or in 
the context of mixed-integer optimal control, see Achtziger and Kanzow [2008], Kirches [2011], Palagachev and Gerdts [2015]. Theoretical and numerical results on problems of type (MPVC) can be found in e.g. Achtziger and Kanzow [2008], Achtziger et al. [2012], Hoheisel [2009], Hoheisel and Kanzow [2007], Hoheisel et al. [2012], Izmailov and Solodov [2009].

Again, we want to transfer (MPVC) into a problem of type (MPDC). Therefore, we define $S_{1}^{\mathrm{VC}}, S_{2}^{\mathrm{VC}} \subset \mathbb{R}^{2}$ by means of $S_{1}^{\mathrm{VC}}:=\mathbb{R}_{+} \times\{0\}$ and $S_{2}^{\mathrm{VC}}:=\mathbb{R}_{-} \times \mathbb{R}_{+}$. Furthermore, we set $\mathcal{J}:=\{1,2\}^{l}$,

$$
\forall \alpha \in \mathcal{J}: \quad D_{\alpha}^{\mathrm{VC}}:=\mathbb{R}_{-}^{p} \times\left\{0^{q}\right\} \times \prod_{j=1}^{l} S_{\alpha_{j}}^{\mathrm{VC}},
$$

as well as $D^{\mathrm{VC}}:=\bigcup_{\alpha \in \mathcal{J}} D_{\alpha}^{\mathrm{VC}}$. Using the function $F$ defined in (13), the feasible set of (MPVC) can be expressed in the compact form $X^{\mathrm{VC}}:=\left\{x \in \mathbb{R}^{n} \mid F(x) \in D^{\mathrm{VC}}\right\}$, see Figure 2. Let us fix a feasible point $\bar{x} \in X^{\mathrm{VC}}$ of (MPVC). We will exploit the index sets
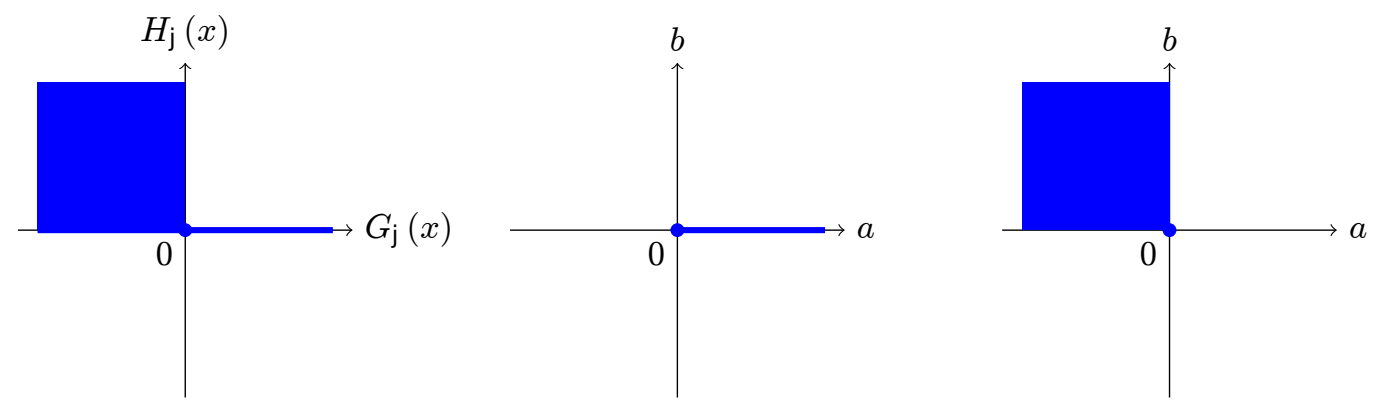

Figure 2: Geometric illustrations of $X^{\mathrm{VC}}$ (left), $S_{1}^{\mathrm{VC}}$ (middle), and $S_{2}^{\mathrm{VC}}$ (right), respectively.

defined below:

$$
\begin{aligned}
I_{+0}(\bar{x}) & :=\left\{j \in\{1, \ldots, l\} \mid H_{j}(\bar{x})>0, G_{j}(\bar{x})=0\right\}, \\
I_{+-}(\bar{x}) & :=\left\{j \in\{1, \ldots, l\} \mid H_{j}(\bar{x})>0, G_{j}(\bar{x})<0\right\}, \\
I_{0+}(\bar{x}) & :=\left\{j \in\{1, \ldots, l\} \mid H_{j}(\bar{x})=0, G_{j}(\bar{x})>0\right\}, \\
I_{0-}(\bar{x}) & :=\left\{j \in\{1, \ldots, l\} \mid H_{j}(\bar{x})=0, G_{j}(\bar{x})<0\right\}, \\
I_{00}(\bar{x}) & :=\left\{j \in\{1, \ldots, l\} \mid H_{j}(\bar{x})=0, G_{j}(\bar{x})=0\right\} .
\end{aligned}
$$

Furthermore, we set $I(\bar{x}):=\left\{\alpha \in \mathcal{J} \mid F(\bar{x}) \in D_{\alpha}^{\mathrm{VC}}\right\}$. Then, we obtain the following characterization for any $\alpha \in \mathcal{J}$ :

$$
\alpha \in I(\bar{x}) \Longleftrightarrow \forall j \in I_{0+}(\bar{x}): \alpha_{j}=1 \wedge \forall j \in I_{+0}(\bar{x}) \cup I_{+-}(\bar{x}) \cup I_{0-}(\bar{x}): \alpha_{j}=2 .
$$

Similar as in Section 5.1, the tangent and Fréchet normal cones to $S_{1}^{\mathrm{VC}}$ and $S_{2}^{\mathrm{VC}}$ can be computed. As a result, one obtains that the constraint qualification MPDC-LICQ takes 
the following form for (MPVC):

$$
\left.\begin{array}{l}
0^{n}=\nabla g(\bar{x})^{\top} \lambda+\nabla h(\bar{x})^{\top} \rho+\nabla G(\bar{x})^{\top} \mu+\nabla H(\bar{x})^{\top} \nu, \\
\forall j \notin I^{g}(\bar{x}): \lambda_{j}=0, \\
\forall j \in I_{+-}(\bar{x}) \cup I_{0+}(\bar{x}) \cup I_{0-}(\bar{x}): \mu_{j}=0, \\
\forall j \in I_{+0}(\bar{x}) \cup I_{+-}(\bar{x}): \nu_{j}=0
\end{array}\right\} \Longrightarrow\left\{\begin{array}{l}
\lambda=0^{p}, \rho=0^{q}, \\
\mu=\nu=0^{l} .
\end{array}\right.
$$

This condition is equivalent to the linear independence of the vectors from

$$
\begin{aligned}
& \left\{\nabla g_{j}(\bar{x}) \mid j \in I^{g}(\bar{x})\right\} \cup\left\{\nabla h_{j}(\bar{x}) \mid j \in\{1, \ldots, q\}\right\} \\
& \quad \cup\left\{\nabla G_{j}(\bar{x}) \mid j \in I_{+0}(\bar{x}) \cup I_{00}(\bar{x})\right\} \cup\left\{\nabla H_{j}(\bar{x}) \mid j \in I_{0+}(\bar{x}) \cup I_{0-}(\bar{x}) \cup I_{00}(\bar{x})\right\}
\end{aligned}
$$

which is referred to as MPVC-LICQ in the literature, see [Hoheisel and Kanzow, 2007, Definition 4.1]. The associated S-stationarity system from Definition 3.9 reads as follows:

$$
\begin{aligned}
& 0^{n}=\nabla f(\bar{x})+\nabla g(\bar{x})^{\top} \lambda+\nabla h(\bar{x})^{\top} \rho+\nabla G(\bar{x})^{\top} \mu+\nabla H(\bar{x})^{\top} \nu, \\
& \lambda \geq 0^{p}, \forall j \notin I^{g}(\bar{x}): \lambda_{j}=0, \\
& \forall j \in I_{+0}(\bar{x}): \mu_{j} \geq 0, \\
& \forall j \in I_{+-}(\bar{x}) \cup I_{0+}(\bar{x}) \cup I_{0-}(\bar{x}) \cup I_{00}(\bar{x}): \mu_{j}=0, \\
& \forall j \in I_{+0}(\bar{x}) \cup I_{+-}(\bar{x}): \nu_{j}=0, \\
& \forall j \in I_{0-}(\bar{x}) \cup I_{00}(\bar{x}): \nu_{j} \leq 0 .
\end{aligned}
$$

We note that this system precisely coincides with the system of strong stationarity for (MPVC) which has been stated in [Hoheisel and Kanzow, 2007, Definition 2.1]. One can easily check that the linearization cone from (2) and the critical cone from (7) equal the respective cones from [Hoheisel and Kanzow, 2007, Section 4]. Thus, our Theorems 3.8, 4.2 and 4.3 precisely recover [Hoheisel and Kanzow, 2009, Corollary 4.5] and [Hoheisel and Kanzow, 2007, Theorems 4.3, 4.4]. Additionally, the stability result from Theorem 4.5 is valid for (MPVC) as well. To the best of our knowledge, this fact cannot be found in the available literature on (MPVC).

\subsection{Application to CCMPs}

Let $\|\cdot\|_{0}: \mathbb{R}^{n} \rightarrow \mathbb{R}$ be the map which assigns to each vector from $\mathbb{R}^{n}$ the number of its nonzero components. For some constant $\kappa \in\{1, \ldots, n-1\}$,

$$
\begin{aligned}
f(x) & \rightarrow \min & & \\
g_{j}(x) & \leq 0 & & j=1, \ldots, p \\
h_{j}(x) & =0 & & j=1, \ldots, q \\
\|x\|_{0} & \leq \kappa & &
\end{aligned}
$$

is a nonlinear so-called cardinality-constrained optimization problem. Problems of the form (CCMP) appear frequently in the context of e.g. compressed sensing or portfolio optimization. Recently, first- and second-order optimality conditions as well as a relaxationbased numerical solution method for (CCMP) were investigated in Bucher and Schwartz 
[2018], Burdakov et al. [2016], Červinka et al. [2016]. The considerations in these papers are based on the surrogate problem

$$
\begin{aligned}
f(x) & \rightarrow \min & & \\
g_{j}(x) & \leq 0 & & j=1, \ldots, p \\
h_{j}(x) & =0 & & j=1, \ldots, q \\
\mathrm{e} \cdot y-(n-\kappa) & \geq 0 & & \\
x_{i} y_{i} & =0 & & i=1, \ldots, n \\
0 \leq y_{i} & \leq 1 & & i=1, \ldots, n
\end{aligned}
$$

which is closely related to (CCMP), see [Burdakov et al., 2016, Section 3] for details. Above, e $\in \mathbb{R}^{n}$ represents the all-ones vector. As suggested in Pan et al. [2017], it is also possible to tackle (CCMP) directly by exploiting a variational analysis approach. Here, we will strike the latter path.

In order to transfer (CCMP) into a program of type (MPDC), let us introduce the index set $\mathcal{J}:=\left\{\alpha \in\{1,2\}^{n} \mid \sum_{i=1}^{n} \alpha_{i}=n+\kappa\right\}$. Now, for each $\alpha \in\{1,2\}^{n}$, we introduce $\mathbb{R}_{\alpha}^{n}:=\operatorname{span}\left\{\mathbf{e}_{i} \mid \alpha_{i}=2\right\}$ where $\mathbf{e}_{i} \in \mathbb{R}^{n}$ denoted the $i$-th unit vector from $\mathbb{R}^{n}$. We set

$$
\forall \alpha \in \mathcal{J}: \quad D_{\alpha}^{\mathrm{C}}:=\mathbb{R}_{-}^{p} \times\left\{0^{q}\right\} \times \mathbb{R}_{\alpha}^{n}
$$

as well as $D^{\mathrm{C}}:=\bigcup_{\alpha \in \mathcal{J}} D_{\alpha}^{\mathrm{C}}$. Furthermore, let us define $F: \mathbb{R}^{n} \rightarrow \mathbb{R}^{p+q+n}$ by means of

$$
\forall x \in \mathbb{R}^{n}: \quad F(x):=\left[\begin{array}{lll}
g(x)^{\top} & h(x)^{\top} \quad x^{\top}
\end{array}\right]^{\top} .
$$

Now, the feasible set of (CCMP) can be represented by $X^{\mathrm{C}}:=\left\{x \in \mathbb{R}^{n} \mid F(x) \in D^{\mathrm{C}}\right\}$. Let us fix a feasible point $\bar{x} \in X^{\mathrm{C}}$ of (CCMP). We will exploit the index sets

$$
I_{ \pm}(\bar{x}):=\left\{i \in\{1, \ldots, n\} \mid x_{i} \neq 0\right\}, \quad I_{0}(\bar{x}):=\{1, \ldots, n\} \backslash I_{ \pm}(\bar{x}) .
$$

Furthermore, we will make use of $I(\bar{x}):=\left\{\alpha \in \mathcal{J} \mid F(\bar{x}) \in D_{\alpha}^{\mathrm{C}}\right\}$. For arbitrary $\alpha \in \mathcal{J}$, we obtain

$$
\alpha \in I(\bar{x}) \Longleftrightarrow \forall i \in I_{ \pm}(\bar{x}): \alpha_{i}=2
$$

Clearly, we have

$$
\forall \alpha \in I(\bar{x}): \quad \mathcal{T}_{\mathbb{R}_{\alpha}^{n}}(\bar{x})=\mathbb{R}_{\alpha}^{n}, \quad \widehat{\mathcal{N}}_{\mathbb{R}_{\alpha}^{n}}(\bar{x})=\mathbb{R}_{3 \mathrm{e}-\alpha}^{n} .
$$

This can be used to compute the tangent and Fréchet normal cone to $D_{\alpha}^{\mathrm{C}}$ for each $\alpha \in I(\bar{x})$. The resulting constraint qualification MPDC-LICQ for (CCMP) takes the form

$$
\left.\begin{array}{l}
0^{n}=\nabla g(\bar{x})^{\top} \lambda+\nabla h(\bar{x})^{\top} \rho+\mu, \\
\forall j \notin I^{g}(\bar{x}): \lambda_{j}=0, \\
\forall i \in I_{ \pm}(\bar{x}): \mu_{i}=0
\end{array}\right\} \Longrightarrow \lambda=0^{p}, \rho=0^{q}, \mu=0^{n}
$$

which is equivalent to the linear independence of the vectors from

$$
\left\{\nabla g_{j}(\bar{x}) \mid j \in I^{g}(\bar{x})\right\} \cup\left\{\nabla h_{j}(\bar{x}) \mid j \in\{1, \ldots, q\}\right\} \cup\left\{\mathrm{e}_{i} \mid i \in I_{0}(\bar{x})\right\},
$$


and the latter is well known as CC-LICQ in the literature, see e.g. [Červinka et al., 2016, Definition 3.11]. The associated system of S-stationarity from Definition 3.9 reads as follows:

$$
\begin{aligned}
& 0^{n}=\nabla f(\bar{x})+\nabla g(\bar{x})^{\top} \lambda+\nabla h(\bar{x})^{\top} \rho+\mu, \\
& \lambda \geq 0^{p}, \forall j \notin I^{g}(\bar{x}): \lambda_{j}=0, \\
& \|\bar{x}\|_{0}=\kappa \Longrightarrow \forall i \in I_{ \pm}(\bar{x}): \mu_{i}=0 \\
& \|\bar{x}\|_{0}<\kappa \Longrightarrow \mu=0^{n} .
\end{aligned}
$$

Defining $\bar{y} \in \mathbb{R}^{n}$ by

$$
\forall i \in\{1, \ldots, n\}: \quad \bar{y}_{i}:= \begin{cases}0 & i \in I_{ \pm}(\bar{x}), \\ 1 & i \in I_{0}(\bar{x}),\end{cases}
$$

we can check that whenever $\bar{x}$ is S-stationary for (CCMP) in the above sense, then $(\bar{x}, \bar{y})$ is a feasible point of (14) which is strongly stationary in the sense of [Burdakov et al., 2016, Definition 4.6] whenever $\|\bar{x}\|_{0}=\kappa$ holds. In case $\|\bar{x}\|_{0}<\kappa$, the S-stationarity conditions from above are more restrictive than the strong stationarity conditions for (14) known from the literature. However, Theorem 3.8 precisely recovers [Bucher and Schwartz, 2018, Proposition 2.1] in the setting at hand.

Some calculations show that the linearization cone from (2) associated with (CCMP) is given by

$$
\mathcal{L}_{X^{\mathrm{C}}}(\bar{x})=\left\{d \in \mathbb{R}^{n} \mid \begin{array}{rlrl}
\nabla g_{j}(\bar{x}) \cdot d & \leq 0 & & j \in I^{g}(\bar{x}) \\
\nabla h_{j}(\bar{x}) \cdot d & =0 & & j \in\{1, \ldots, q\} \\
\left|\left\{i \in I_{0}(\bar{x}) \mid d_{i}=0\right\}\right| & \geq n-\kappa &
\end{array}\right\} .
$$

Whenever $\bar{x}$ is an S-stationary point of (CCMP), then due to Lemma 4.1, for each corresponding multiplier $(\lambda, \rho, \mu) \in \mathbb{R}^{p} \times \mathbb{R}^{q} \times \mathbb{R}^{n}$ which solves the system of S-stationarity, the associated critical cone from (7) is given by

$$
\mathcal{C}_{X^{\mathrm{C}}}(\bar{x})=\left\{d \in \mathbb{R}^{n} \mid \begin{array}{rlrl}
\nabla g_{j}(\bar{x}) \cdot d & \leq 0 & & j \in I^{g}(\bar{x}), \lambda_{j}=0 \\
\nabla g_{j}(\bar{x}) \cdot d & =0 & & j \in I^{g}(\bar{x}), \lambda_{j}>0 \\
\nabla h_{j}(\bar{x}) \cdot d & =0 & & j \in\{1, \ldots, q\} \\
\left|\left\{i \in I_{0}(\bar{x}) \mid d_{i}=0\right\}\right| & \geq n-\kappa &
\end{array}\right\} .
$$

Thus, in the context of (CCMP), Theorem 4.2 precisely recovers [Bucher and Schwartz, 2018, Corollary 3.1] and [Pan et al., 2017, Theorem 4.1] while the statement of Theorem 4.3 parallels [Bucher and Schwartz, 2018, Corollary 3.2] and enhances [Pan et al., 2017, Theorem 4.2]. The stability result from Theorem 4.5 can be found in slightly enhanced form in [Bucher and Schwartz, 2018, Corollary 3.3]. 


\subsection{Application to MPSCs}

Let us consider so-called mathematical programs with switching constraints which are optimization problems of the form

$$
\begin{aligned}
f(x) & \rightarrow \min & & \\
g_{j}(x) & \leq 0 & & j=1, \ldots, p \\
h_{j}(x) & =0 & & j=1, \ldots, q \\
G_{j}(x) \cdot H_{j}(x) & =0 & & j=1, \ldots, l .
\end{aligned}
$$

Models of type (MPSC) arise from the discretization of so-called switching-constrained optimal control problems, see e.g. Clason et al. [2017] and the references therein, as well as the reformulation of logical or-constraints, see [Mehlitz, 2019, Section 7], or semicontinuity conditions on variables, see [Kanzow et al., 2018, Section 5.2.3]. First-order necessary optimality conditions as well as numerical relaxation methods for problems of type (MPSC) can be found in Kanzow et al. [2018], Mehlitz [2019].

Let us transfer (MPSC) into a program of type (MPDC). Therefore, we introduce $S_{1}^{\mathrm{SC}}:=\mathbb{R} \times\{0\}$ and $S_{2}^{\mathrm{SC}}:=\{0\} \times \mathbb{R}$ as well as $\mathcal{J}:=\{1,2\}^{l}$. We set

$$
\forall \alpha \in \mathcal{J}: \quad D_{\alpha}^{\mathrm{SC}}:=\mathbb{R}_{-}^{p} \times\left\{0^{q}\right\} \times \prod_{j=1}^{l} S_{\alpha_{j}}^{\mathrm{SC}}
$$

as well as $D^{\mathrm{SC}}:=\bigcup_{\alpha \in \mathcal{J}} D_{\alpha}^{\mathrm{SC}}$. Using the mapping $F$ defined in (13), the feasible set of (MPSC) can be represented by $X^{\mathrm{SC}}:=\left\{x \in \mathbb{R}^{n} \mid F(x) \in D^{\mathrm{SC}}\right\}$. The variational geometry of $X^{\mathrm{SC}}$ is visualized in Figure 3. For a feasible point $\bar{x} \in X^{\mathrm{SC}}$ of (MPSC), we
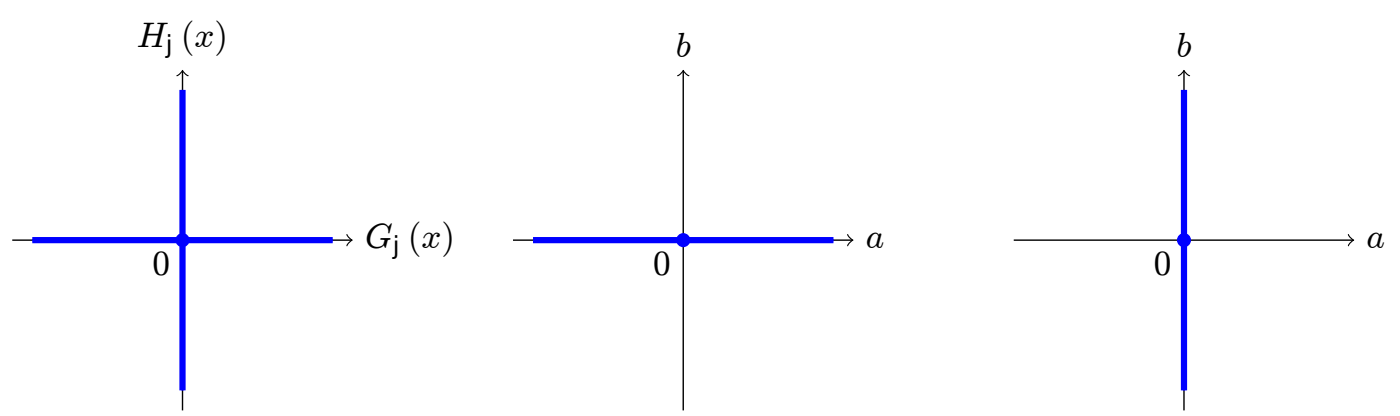

Figure 3: Geometric illustrations of $X^{\mathrm{SC}}$ (left), $S_{1}^{\mathrm{SC}}$ (middle), and $S_{2}^{\mathrm{SC}}$ (right), respectively.

introduce the following index sets:

$$
\begin{aligned}
I^{G}(\bar{x}) & :=\left\{j \in\{1, \ldots, l\} \mid G_{j}(\bar{x})=0, H_{j}(\bar{x}) \neq 0\right\}, \\
I^{H}(\bar{x}) & :=\left\{j \in\{1, \ldots, l\} \mid G_{j}(\bar{x}) \neq 0, H_{j}(\bar{x})=0\right\}, \\
I^{G H}(\bar{x}) & :=\left\{j \in\{1, \ldots, l\} \mid G_{j}(\bar{x})=0, H_{j}(\bar{x})=0\right\} .
\end{aligned}
$$

Furthermore, we set $I(\bar{x}):=\left\{\alpha \in \mathcal{J} \mid F(\bar{x}) \in D_{\alpha}^{\mathrm{SC}}\right\}$. Thus, we have

$$
\alpha \in I(\bar{x}) \Longleftrightarrow \forall j \in I^{G}(\bar{x}): \alpha_{j}=2 \wedge \forall j \in I^{H}(\bar{x}): \alpha_{j}=1
$$


for each $\alpha \in \mathcal{J}$. Computing the tangent and Fréchet normal cones to $S_{1}^{\mathrm{SC}}$ and $S_{2}^{\mathrm{SC}}$, we obtain that the constraint qualification MPDC-LICQ takes the following form for (MPSC):

$$
\left.\begin{array}{l}
0^{n}=\nabla g(\bar{x})^{\top} \lambda+\nabla h(\bar{x})^{\top} \rho+\nabla G(\bar{x})^{\top} \mu+\nabla H(\bar{x})^{\top} \nu, \\
\forall j \notin I^{g}(\bar{x}): \lambda_{j}=0, \\
\forall j \in I^{H}(\bar{x}): \mu_{j}=0, \\
\forall j \in I^{G}(\bar{x}): \nu_{j}=0
\end{array}\right\} \Longrightarrow\left\{\begin{array}{l}
\lambda=0^{p}, \rho=0^{q}, \\
\mu=\nu=0^{l} .
\end{array}\right.
$$

We note that this is equivalent to the linear independence of all the vectors from

$$
\begin{aligned}
& \left\{\nabla g_{j}(\bar{x}) \mid j \in I^{g}(\bar{x})\right\} \cup\left\{\nabla h_{j}(\bar{x}) \mid j \in\{1, \ldots, q\}\right\} \\
& \cup\left\{\nabla G_{j}(\bar{x}) \mid j \in I^{G}(\bar{x}) \cup I^{G H}(\bar{x})\right\} \cup\left\{\nabla H_{j}(\bar{x}) \mid j \in I^{H}(\bar{x}) \cup I^{G H}(\bar{x})\right\}
\end{aligned}
$$

and this condition is called MPSC-LICQ in the literature, see [Mehlitz, 2019, Definition 4.4]. The associated S-stationarity system from Definition 3.9 is given by

$$
\begin{aligned}
& 0^{n}=\nabla f(\bar{x})+\nabla g(\bar{x})^{\top} \lambda+\nabla h(\bar{x})^{\top} \rho+\nabla G(\bar{x})^{\top} \mu+\nabla H(\bar{x})^{\top} \nu, \\
& \lambda \geq 0^{p}, \forall j \notin I^{g}(\bar{x}): \lambda_{j}=0, \\
& \forall j \in I^{H}(\bar{x}) \cup I^{G H}(\bar{x}): \mu_{j}=0, \\
& \forall j \in I^{G}(\bar{x}) \cup I^{G H}(\bar{x}): \nu_{j}=0
\end{aligned}
$$

and equals the problem-tailored system of strong stationarity as it has been stated in [Mehlitz, 2019, Definition 4.3]. The results of Theorem 3.8 can be found in [Mehlitz, 2019, Theorem 4.5].

One can easily check that the linearization cone from (2) possesses the form

$$
\mathcal{L}_{X^{\mathrm{SC}}}(\bar{x})=\left\{d \in \mathbb{R}^{n} \mid \begin{array}{rl}
\nabla g(\bar{x}) \cdot d \leq 0 & j \in I^{g}(\bar{x}) \\
\nabla h(\bar{x}) \cdot d=0 & j \in\{1, \ldots, q\} \\
\nabla G(\bar{x}) \cdot d=0 & j \in I^{G}(\bar{x}) \\
\nabla H(\bar{x}) \cdot d=0 & j \in I^{H}(\bar{x}) \\
(\nabla G(\bar{x}) \cdot d)(\nabla H(\bar{x}) \cdot d)=0 & j \in I^{G H}(\bar{x})
\end{array}\right\}
$$

while we obtain

$$
\mathcal{C}_{X^{\mathrm{SC}}}(\bar{x})=\left\{d \in \mathbb{R}^{n} \mid \begin{array}{rl}
\nabla g(\bar{x}) \cdot d \leq 0 & j \in I^{g}(\bar{x}), \lambda_{j}=0 \\
\nabla g(\bar{x}) \cdot d=0 & j \in I^{g}(\bar{x}), \lambda_{j}>0 \\
\nabla h(\bar{x}) \cdot d=0 & j \in\{1, \ldots, q\} \\
\nabla G(\bar{x}) \cdot d=0 & j \in I^{G}(\bar{x}) \\
\nabla H(\bar{x}) \cdot d=0 & j \in I^{H}(\bar{x}) \\
(\nabla G(\bar{x}) \cdot d)(\nabla H(\bar{x}) \cdot d)=0 & j \in I^{G H}(\bar{x})
\end{array}\right\}
$$


for the associated critical cone provided $\bar{x}$ is an S-stationary point of (MPSC) with associated multipliers $(\lambda, \rho, \mu, \nu) \in \mathbb{R}^{p} \times \mathbb{R}^{q} \times \mathbb{R}^{l} \times \mathbb{R}^{l}$, see Lemma 4.1. Based on this critical cone, Theorems 4.2 and 4.3 provide a necessary and sufficient second-order optimality condition for (MPSC). Furthermore, Theorem 4.5 yields a criterion which ensures local uniqueness of S-stationary points associated with (MPSC). To the best of our knowledge, these are new results on the problem class (MPSC).

\section{Final remarks}

In this paper, we introduced a reasonable abstract version of the prominent linear independence constraint qualification which applies to mathematical programs with disjunctive constraints. We were able to derive first- and second-order optimality conditions based on strongly stationary points under validity of this constraint qualification in elementary way. Finally, we applied our findings to several different instances of disjunctive programs in order to underline that this new constraint qualification is reasonable. By means of switching-constrained mathematical problems, it has been demonstrated that our theory does not only recover well-known results from the literature but can be used to infer new results on specific instances of disjunctive programming as well.

\section{Acknowledgments}

We would like to thank two anonymous reviewers for several valuable comments and remarks which led to a significant improvement of this paper during revision. Particularly, we are in debt to one of the referees for suggesting the current version of Lemma 2.3 as well as its proof.

\section{References}

W. Achtziger and C. Kanzow. Mathematical programs with vanishing constraints: optimality conditions and constraint qualifications. Mathematical Programming, Series A, 114(1):69-99, 2008. doi: 10.1007/s10107-006-0083-3.

W. Achtziger, C. Kanzow, and T. Hoheisel. On a relaxation method for mathematical programs with vanishing constraints. GAMM-Mitteilungen, 35(2):110-130, 2012. doi: 10.1002/gamm.201210009.

J.-P. Aubin and H. Frankowska. Set-valued Analysis. Modern Birkhäuser Classics. Birkhäuser, Boston, 2009. Reprint of the 1990 edition.

M. Benko and H. Gfrerer. On estimating the regular normal cone to constraint systems and stationarity conditions. Optimization, 66(1):61-92, $2017 . \quad$ doi: 10.1080/02331934.2016.1252915. 
M. Benko and H. Gfrerer. New verifiable stationarity concepts for a class of mathematical programs with disjunctive constraints. Optimization, 67(1):1-23, 2018. doi: 10.1080/02331934.2017.1387547.

J. F. Bonnans and A. Shapiro. Perturbation Analysis of Optimization Problems. Springer, New York, 2000.

M. Bucher and A. Schwartz. Second-Order Optimality Conditions and Improved Convergence Results for Regularization Methods for Cardinality-Constrained Optimization Problems. Journal of Optimization Theory and Applications, 178(2):383-410, 2018. doi: 10.1007/s10957-018-1320-7.

O. P. Burdakov, C. Kanzow, and A. Schwartz. Mathematical programs with cardinality constraints: reformulation by complementarity-type conditions and a regularization method. SIAM Journal on Optimization, 26(1):397-425, 2016. doi: $10.1137 / 140978077$.

M. Červinka, C. Kanzow, and A. Schwartz. Constraint qualifications and optimality conditions for optimization problems with cardinality constraints. Mathematical Programming, Series A, 160(1):353-377, 2016. doi: 10.1007/s10107-016-0986-6.

C. Christof and G. Wachsmuth. No-Gap Second-Order Conditions via a Directional Curvature Functional. SIAM Journal on Optimization, 28(3):2097-2130, 2018. doi: 10.1137/17M1140418.

C. Clason, A. Rund, and K. Kunisch. Nonconvex penalization of switching control of partial differential equations. Systems \& Control Letters, 106:1-8, 2017. doi: 10.1016/j.sysconle.2017.05.006.

M. L. Flegel, C. Kanzow, and J. V. Outrata. Optimality conditions for disjunctive programs with application to mathematical programs with equilibrium constraints. Set-Valued Analysis, 15(2):139-162, 2007. doi: 10.1007/s11228-006-0033-5.

H. Gfrerer. On Directional Metric Subregularity and Second-Order Optimality Conditions for a Class of Nonsmooth Mathematical Programs. SIAM Journal on Optimization, 23(1):632-665, 2013. doi: 10.1137/120891216.

H. Gfrerer. Optimality Conditions for Disjunctive Programs Based on Generalized Differentiation with Application to Mathematical Programs with Equilibrium Constraints. SIAM Journal on Optimization, 24(2):898-931, 2014. doi: 10.1137/130914449.

H. Gfrerer and B. Mordukhovich. Complete Characterizations of Tilt Stability in Nonlinear Programming under Weakest Qualification Conditions. SIAM Journal on Optimization, 25(4):2081-2119, 2015. doi: 10.1137/15M1012608.

H. Gfrerer and J. V. Outrata. On Computation of Generalized Derivatives of the NormalCone Mapping and Their Applications. Mathematics of Operations Research, 41(4): 1535-1556, 2016. doi: 10.1287/moor.2016.0789. 
L. Guo, G.-H. Lin, and J. J. Ye. Second-Order Optimality Conditions for Mathematical Programs with Equilibrium Constraints. Journal of Optimization Theory and Applications, 158(1):33-64, 2013. doi: 10.1007/s10957-012-0228-x.

R. Henrion and J. V. Outrata. Calmness of constraint systems with applications. Mathematical Programming, 104(2):437-464, 2005. doi: 10.1007/s10107-005-0623-2.

T. Hoheisel. Mathematical Programs with Vanishing Constraints. PhD thesis, University of Würzburg, 2009.

T. Hoheisel and C. Kanzow. First- and second-order optimality conditions for mathematical programs with vanishing constraints. Applications of Mathematics, 52(6):495-514, 2007. doi: 10.1007/s10492-007-0029-y.

T. Hoheisel and C. Kanzow. On the Abadie and Guignard constraint qualifications for mathematical programmes with vanishing constraints. Optimization, 58(4):431-448, 2009. doi: $10.1080 / 02331930701763405$.

T. Hoheisel, C. Kanzow, and A. Schwartz. Convergence of a local regularization approach for mathematical programmes with complementarity or vanishing constraints. Optimization Methods and Software, 27(3):483-512, 2012. doi: 10.1080/10556788.2010.535170.

T. Hoheisel, C. Kanzow, and A. Schwartz. Theoretical and numerical comparison of relaxation methods for mathematical programs with complementarity constraints. Mathematical Programming, 137(1):257-288, 2013. doi: 10.1007/s10107-011-0488-5.

A. F. Izmailov and M. V. Solodov. Mathematical Programs with Vanishing Constraints: Optimality Conditions, Sensitivity, and a Relaxation Method. Journal of Optimization Theory and Applications, 142(3):501-532, 2009. doi: 10.1007/s10957-009-9517-4.

C. Kanzow, P. Mehlitz, and D. Steck. Relaxation schemes for mathematical programs with switching constraints. Preprint arXiv, 2018. URL https://arxiv.org/abs/1809.02388.

C. Kirches. Fast Numerical Methods for Mixed-Integer Nonlinear Model-Predictive Control. PhD thesis, University of Heidelberg, 2011.

Z.-Q. Luo, J.-S. Pang, and D. Ralph. Mathematical Programs with Equilibrium Constraints. Cambridge University Press, Cambridge, 1996.

P. Mehlitz. Stationarity conditions and constraint qualifications for mathematical programs with switching constraints. Mathematical Programming, pages 1-38, 2019. doi: 10.1007/s10107-019-01380-5.

J. V. Outrata, M. Kočvara, and J. Zowe. Nonsmooth Approach to Optimization Problems with Equilibrium Constraints. Kluwer Academic, Dordrecht, 1998. 
K. Palagachev and M. Gerdts. Mathematical Programs with Blocks of Vanishing Constraints Arising in Discretized Mixed-Integer Optimal Control Problems. Set-Valued and Variational Analysis, 23(1):149-167, 2015. doi: 10.1007/s11228-014-0297-0.

L. Pan, N. Xiu, and J. Fan. Optimality conditions for sparse nonlinear programming. Science China Mathematics, 60(5):759-776, 2017. doi: 10.1007/s11425-016-9010-x.

J. Penot. Second-Order Conditions for Optimization Problems with Constraints. SIAM Journal on Control and Optimization, 37(1):303-318, 1998. doi: 10.1137/S0363012996311095.

R. T. Rockafellar and R. J.-B. Wets. Variational Analysis, volume 317 of Grundlehren der mathematischen Wissenschaften. Springer, Berlin, 1998.

S. Scheel and S. Scholtes. Mathematical programs with complementarity constraints: Stationarity, optimality, and sensitivity. Mathematics of Operations Research, 25(1): 1-22, 2000. doi: 10.1287/moor.25.1.1.15213.

S. Scholtes. Nonconvex Structures in Nonlinear Programming. Operations Research, 52 (3):368-383, 2004. doi: 10.1287/opre.1030.0102.

J. J. Ye. Necessary and sufficient optimality conditions for mathematical programs with equilibrium constraints. Journal of Mathematical Analysis and Applications, 307(1): 350 - 369, 2005. doi: 10.1016/j.jmaa.2004.10.032. 